\title{
Beiträge zur Biochemie der Mikroorganismen.
}

Von

\author{
Hartwig Franzen.
}

III. Mitteilung.

Über die Vergärung der Ameisenøäure durch Bacillus Phlymouthensis.

Von

Hartwig Franzen und G. Greve.

(Mitteilung aus dem chemischen Institut der Universität Heidelberg.)

(Der Redaktion zugegangen am 20. Juni 1910.)

Die theoretischen Erörterungen zu dieser Abhandlung sind schon in der zweiten Mitteilung ${ }^{1}$ ) gegeben worden, soda $B$ sich nur noch erübrigt, das nötige Zahlenmaterial mitzuteilen.

\section{A. Stamm Kràl.}

a) Calciumformiat.

1. $27^{\circ}$.

Tabelle Nr. 33.

$0,4602 \mathrm{~g}$ Ameisensäure als Natriumformiat bei $27^{\circ}$.

Bakterium: Bac. Plymouthensis Kràl.

\begin{tabular}{c|c|c|c|c|c}
\hline $\begin{array}{c}\text { Zeit } \\
\text { in } \\
\text { Tagen }\end{array}$ & $\mathbf{g}$ & $\begin{array}{c}\text { Kalomel } \\
\text { HCOOH } \\
\text { gorhandene }\end{array}$ & $\begin{array}{c}\text { Noch } \\
\text { vorhandene } \\
\text { HCOOH } \\
\%\end{array}$ & $\begin{array}{c}\text { Vergoren } \\
\text { HCOOH } \\
\mathbf{g}\end{array}$ & $\begin{array}{c}\text { Vergoren } \\
\text { HCOOH } \\
\%\end{array}$ \\
\hline 1 & 4,7016 & 0,4595 & 99,84 & 0,0007 & 0,13 \\
2 & 4,4548 & 0,4354 & 94,60 & 0,0248 & 5,40 \\
3 & 4,3374 & 0,4239 & 92,11 & 0,0363 & 7,89 \\
4 & 4,2940 & 0,4196 & 91,39 & 0,0406 & 8,61
\end{tabular}

1) Diese Zeitschrift, Bd. LXIV, S. 169 (1910). 
Tabelle Nr. 34.

$0,4602 \mathrm{~g}$ Ameisensäure als Natriumformiat bei $27^{\circ}$.

Bakterium: Bac. Plymouthensis Kràl.

\begin{tabular}{c|c|c|c|c|c}
\hline \hline $\begin{array}{c}\text { Zeit } \\
\text { in } \\
\text { Tagen }\end{array}$ & $\mathrm{g}$ & $\begin{array}{c}\text { Kalomel } \\
\mathrm{gCOOH} \\
\mathrm{g}\end{array}$ & $\begin{array}{c}\text { Noch } \\
\text { vorhandene } \\
\text { HCOOH } \\
\text { \% }\end{array}$ & $\begin{array}{c}\text { Noch } \\
\text { vorhandene } \\
\mathrm{HCOOH} \\
\mathrm{g}\end{array}$ & $\begin{array}{c}\text { Vergoren } \\
\mathrm{HCOOH} \\
\%\end{array}$ \\
\hline 1 & 4,7033 & 0,4596 & 99,88 & 0,0006 & 0,12 \\
2 & 4,4472 & 0,4346 & 94,44 & 0,0256 & 5,66 \\
3 & 4,2396 & 0,4231 & 91,94 & 0,0371 & 8,06
\end{tabular}

Die Neutralisation der Nährbouillon wurde mit Hilfe von Lackmuspapier als Indikator durchgeführt; die Kolben wurden nach Methode 1 geimpft.

Was, die makroskopischen Erscheinungen anbelangt, so war eine Farbstoffbildung überhaupt nicht $z u$ bemerken; schon nach 1 Tag war ein erheblicher Niederschlag von Calciumcarbonat, der sich am folgenden Tage noch vermehrte, zu bemerken.

Vergleich der gefundenen relativen Werte.

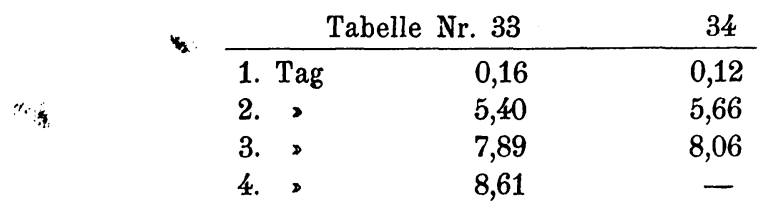

Mittlere Tabelle.

1. Tag

0,14

2. $>$

5,53

3. $>$

7,98

4. >

8,61

Abweichung der gefundenen von den mittleren Werten.

\begin{tabular}{|c|c|c|}
\hline \multicolumn{2}{|c|}{ Tabelle Nr. 33} & 34 \\
\hline 1. $\mathrm{Tag}$ & $+0,02$ & $-0,02$ \\
\hline 2. $\gg$ & $-0,13$ & $+0,13$ \\
\hline 3. » & $-0,09$ & $+0,08$ \\
\hline 4. \ & $\pm 0,00$ & - \\
\hline
\end{tabular}

Die Abweichungen der gefundenen von den mittleren Werten sind recht klein, sie schwanken zwischen $+0,13 \%$ und $-0,13 \%$. 
Beiträge zur Biochemie der Mikroorganismen. III.

Menge der während der einzelnen Tage vergorenen Ameisensäure.

$\begin{array}{cr}\text { Innerhalb des } & \text { sind vergoren } \\ \text { 1. Tages } & 0,14 \\ \text { 2. } & 5,39 \\ \text { 3. } & 2,45 \\ \text { 4. } & 0,63\end{array}$

Vergleich der gefundenen absoluten Werte.

\begin{tabular}{|c|c|c|}
\hline \multicolumn{2}{|c|}{ Tabelle Nr. 33} & 34 \\
\hline 1. Tag & 7 & 6 \\
\hline 2. $>$ & 248 & 256 \\
\hline 3. & 363 & 371 \\
\hline 4. & 406 & - \\
\hline
\end{tabular}

$\begin{array}{lr}\text { 1. Tag } & 7 \\ 2 . \text {, } & 252 \\ 3 . \text {. } & 367 \\ 4 . \text {. } & 406\end{array}$

Abweichung der gefundenen von den mittleren Werten.

Tabelle Nr. 33

\begin{tabular}{lll}
\hline 1. Tag & \pm 0 & -1 \\
$2 .:$ & -4 & +4 \\
$3 .:$ & -4 & +4 \\
$4 .:$ & \pm 0 & -
\end{tabular}

Die Abweichungen der gefundenen von den mittleren Werten sind recht klein, sie schwanken zwischen $+0,0004 \mathrm{~g}$ und $-0,0004 \mathrm{~g}$.

Menge der während der einzelnen Tage vergorenen Ameisensăure.

$\begin{array}{cr}\text { Innerhalb des } & \text { sind vergoren } \\ \text { 1. Tages } & 7 \\ \text { 2. } & 245 \\ \text { 3. } & 115 \\ \text { 4. } & 39\end{array}$

In 4 Tagen werden $0,0406 \mathrm{~g}=8,61 \%$ Ameisensäure vergoren; die größte Gärungsintensität liegt innerhalb des 2. Tages; innerhalb dieses Tages werden $0,0245=5,39 \%$ Ameisensäure vergoren; die Gärung ist nach 4 Tagen noch nicht beendet. Merkwürdig ist, daß innerhalb des 1 . Tages so wenig Ameisensäure vergoren wird. 
2. $37^{\circ}$.

Tabelle Nr. 35.

$0,4602 \mathrm{~g}$ Ameisensäure als Calciumformiat bei $37^{\circ}$.

Bakterium: Bac. Plymouthensis Kràl.

\begin{tabular}{c|c|c|c|c|c}
\hline $\begin{array}{c}\text { Zeit } \\
\text { in } \\
\text { Tagen }\end{array}$ & Kalomel & $\begin{array}{c}\text { Noch } \\
\text { vorhandene } \\
\text { HCOOH } \\
\mathrm{g}\end{array}$ & $\begin{array}{c}\text { Noch } \\
\text { vorhandene } \\
\mathrm{HCOOH} \\
\mathbf{0}\end{array}$ & $\begin{array}{c}\text { Vergoren } \\
\mathrm{HCOOH} \\
\mathrm{g}\end{array}$ & $\begin{array}{c}\text { Vergoren } \\
\mathrm{HCOOH} \\
\%\end{array}$ \\
\hline $\mathbf{1}$ & $\mathbf{4 , 7 1 1 4}$ & 0,4604 & 100,05 & 0,0002 & $+0,05$ \\
$\mathbf{2}$ & $\mathbf{4 , 3 1 6 2}$ & 0,4218 & 91,66 & 0,0384 & $-8,34$ \\
3 & 4,3255 & 0,4227 & 91,85 & 0,0375 & 8,15 \\
4 & 4,3274 & 0,4229 & 91,89 & 0,0373 & 8,11 \\
5 & 4,3206 & 0,4222 & 91,75 & 0,0380 & 8,25
\end{tabular}

Tabelle Nr. 36.

$0,4602 \mathrm{~g}$ Ameisensäure als Calciumformiat bei $37^{\circ}$.

Bakterium: Bac. Plymouthensis Kräl.

\begin{tabular}{c|c|c|c|c|c}
\hline $\begin{array}{c}\text { Zeit } \\
\text { in } \\
\text { Tagen }\end{array}$ & $\begin{array}{c}\text { Kalomel } \\
\mathbf{g}\end{array}$ & $\begin{array}{c}\text { voch } \\
\text { vorhandene } \\
\text { HCOOH } \\
\mathbf{g}\end{array}$ & $\begin{array}{c}\text { Noch } \\
\text { vorhandene } \\
\text { HCOOH } \\
\%\end{array}$ & $\begin{array}{c}\text { Vergoren } \\
\text { HCOOH } \\
\mathbf{g}\end{array}$ & $\begin{array}{c}\text { Vergoren } \\
\text { HCOOH } \\
\%\end{array}$ \\
\hline $\mathbf{1}$ & $\mathbf{4} 7098$ & 0,4603 & 100,01 & 0,0001 & $+0,01$ \\
2 & 4,3218 & 0,4224 & 91,77 & 0,0378 & 8,23 \\
3 & $\mathbf{4 , 3 3 0 6}$ & 0,4232 & 91,96 & 0,0370 & 8,04 \\
$\mathbf{4}$ & $\mathbf{4 , 3 1 9 6}$ & 0,4221 & 91,73 & 0,0381 & 8,27 \\
$\mathbf{5}$ & $\mathbf{4 , 3 1 3 8}$ & 0,4216 & 91,61 & 0,0386 & 8,39
\end{tabular}

Die Neutralisation der Bouillon wurde mit Hilfe von Lackmuspapier als Indikator durchgeführt; die Kolben wurden nach Methode 1 geimpft.

Was die makroskopischen Erscheinungen anbelangt, so war Farbstoff bildung nicht zu bemerken; am Boden des Kolbens war ein dicker Niederschlag von Calciumcarbonat vorhanden.

Vergleich der gefundenen relativen Werte.

\begin{tabular}{|c|c|c|}
\hline \multicolumn{2}{|c|}{ Tabelle Nr. 35} & 36 \\
\hline 1. Tag & $+0,05$ & $+0,01$ \\
\hline 2. $\gg$ & $-8,34$ & $-8,23$ \\
\hline 3. » & 8,15 & 8,04 \\
\hline 4. $>$ & 8,11 & 8,27 \\
\hline 5. $\gg$ & 8,25 & 8,39 \\
\hline
\end{tabular}


Mittlere Tabelle.
1. Tag
$+0,03$
2. ,
$-8,29$
3. »
8,10
$4 . \$$
8,19
5. ,
8,32

Abweichung der gefundenen von den mittleren Werten.

\begin{tabular}{lcc}
\multicolumn{2}{c}{ Tabelle Nr. 35 } & 36 \\
\hline 1. Tag & $+0,02$ & $-0,02$ \\
2. & $+0,05$ & $-0,06$ \\
3. & $+0,05$ & $-0,06$ \\
4. & $-0,08$ & $+0,08$ \\
5. & $-0,07$ & $+0,07$
\end{tabular}

Die Abweichungen der gefundenen von den mittleren Werten sind sehr klein, sie schwanken zwischen $+0,08^{\circ} !_{0}$ und $-0,08 \%$.

Menge der während der einzelnen Tage vergorenen

Ameisensäure.

(Berechnet aus der mittleren Tabelle.)

Innerhalb des

1. Tages

2. 》

3. >

4. ,

5. , sind vergoren $\mathrm{HCOOH}$

$$
\begin{array}{r}
+0,03 \\
-8,32 \\
+0,19 \\
-0,09 \\
-0,13
\end{array}
$$

Vergleich der gefundenen absoluten Werte.

\begin{tabular}{rrr} 
& Tabelle Nr. 35 & \multicolumn{1}{c}{36} \\
\hline 1. Tag & +2 & +1 \\
$2 .>$ & -384 & -378 \\
$3 .>$ & 375 & 370 \\
$4 .>$ & 373 & 381 \\
$5 .>$ & 380 & 386
\end{tabular}

Mittlere Tabelle.

$\begin{array}{lr}\text { 1. Tag } & +2 \\ \text { 2. } & 381 \\ \text { 3. } & 373 \\ \text { 4. } & 377 \\ \text { 5. } & 383\end{array}$


Abweichung der gefundenen von den mittleren Werten.

\begin{tabular}{rrr} 
& Tabelle Nr. 35 & \multicolumn{1}{c}{36} \\
\hline 1. Tag & \pm 0 & -1 \\
$2 .:$ & +3 & -3 \\
$3 .:$ & +2 & -3 \\
$4 .:$ & -4 & +4 \\
5. & -3 & +3
\end{tabular}

Die Abweichungen der gefundenen von den mittleren Werten sind sehr klein, sie schwanken zwischen $+0,004 \mathrm{~g}$ und $0,004 \mathrm{~g}$.

Menge der während der einzelnen Tage vergorenen

Ameisensäure.

(Berechnet aus der mittleren Tabelle.)

Innerhalb des

1. Tages

2. $\gg$

3. >

4. $\$$

5. $>$ sind vergoren $\mathrm{HCOOH}$

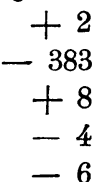

In fünf Tagen werden $0,0383=8,32 \%$ Ameisensäure vergoren. Die größte Gärungsintensität liegt innerhalb des 2. Tages; innerhalb dieses Tages werden $0,0383 \mathrm{~g}=8,32 \%$ Ameisensäure vergoren. Nach dem zweiten Tage ist die Gärung beendet, die an den folgenden Tagen erhaltenen Zahlen zeigen gegenüber denen des 2. Tages nur sehr geringe Unterschiede. Innerhalb des 1. Tages wird überhaupt keine Ameisensäure vergoren.

Vergleich der bei den verschiedenen Temperaturen erhaltenen Zahlen.

Bei beiden Temperaturen liegt die größte Gärungsintensität innerhalb des 2. Tages; bei $27^{\circ}$ wird innerhalb dieses Tages $0,0245 \mathrm{~g}=5,39 \%$ Ameisensäure vergoren, bei $37^{\circ} 0,0383 \mathrm{~g}$ $=8,32 \%$. Während bei $37^{\circ}$ die Gärung nach 2 Tagen beendet ist, ist sie bei $27^{\circ}$ nach 4 Tagen noch nicht beendet. Bei $27^{\circ}$ wird etwas mehr Ameisensäure vergoren als bei $37^{\circ}$; die Unterschiede sind aber nur sehr geringfügig. 
Beiträge zur Biochemie der Mikroorganismen. III.

B) Natriumformiat.

1. $17^{\circ}$.

Tabelle Nr. 37.

0,4602 g Ameisensäure als Natriumformiat bei $17^{\circ}$.

Bakterium: Bac. Plymouthensis Kràl.

\begin{tabular}{c|c|c|c|c|c|c}
\hline \hline $\begin{array}{c}\text { Zeit } \\
\text { in } \\
\text { Tagen }\end{array}$ & $\begin{array}{c}\text { Kolben } \\
\text { Nr. }\end{array}$ & Kalomel & $\begin{array}{c}\text { Noch } \\
\text { vorhandene } \\
\text { HCOOH } \\
\mathbf{g}\end{array}$ & $\begin{array}{c}\text { Noch } \\
\text { vorhandene } \\
\text { HCOOH } \\
\%\end{array}$ & $\begin{array}{c}\text { Vergoren } \\
\text { HCOOH } \\
\mathrm{g}\end{array}$ & $\begin{array}{c}\text { Vergoren } \\
\text { HCOOH } \\
\%\end{array}$ \\
\hline 1 & 1 & 4,5002 & 0,4398 & 95,56 & 0,0204 & 4,44 \\
2 & 2 & 4,1176 & 0,4024 & 87,44 & 0,0578 & 12,56 \\
3 & - & - & - & - & - & - \\
4 & 3 & 4,0254 & 0,3934 & 85,48 & 0,0668 & 14,52 \\
5 & 4 & 3,6014 & 0,3520 & 76,48 & 0,1082 & 23,52 \\
6 & 5 & 3,2636 & 0,3189 & 69,31 & 0,1413 & 30,69 \\
7 & 6 & 3,0846 & 0,3015 & 65,50 & 0,1587 & 34,50 \\
8 & 7 & 2,9630 & 0,2896 & 62,92 & 0,1706 & 37,08 \\
9 & 8 & 2,8954 & 0,2830 & 61,49 & 0,1772 & 38,51 \\
10 & 9 & 2,8197 & 0,2756 & 59,88 & 0,1846 & 40,12
\end{tabular}

Die Neutralisation der Bouillon wurde mit Hilfe von

Phenolphthalein als Indikator durchgeführt; die Kolben wurden nach Methode 1 geimpft.

Makroskopische Erscheinungen:

Nach 1 Tag: Schwache Trübung, Ansätze zur Hautbildung scheinen vorhanden zu sein, kein Farbstoff, kein Bodensatz; Kolben sehen gleichmäßig aus.

Nach 2 Tagen: Trübung etwas stärker als am Tage vorher, Hautbildung nicht fortgeschritten, an der Wandung des Glases, dort wo die Flüssigkeit aufhört, einige schwach rosenrote Partien, kein Bodensatz; Kolben sehen gleichmäßig aus.

Nach 3 Tagen: Ziemlich starke Trübung, Kolben 4 und 6 etwas stärker getrübt als $5,7,8,9$, auf Kolben 4 und 6 wenig rosenrote nicht zusammenhängende Haut, auf den anderen Kolben keine Haut, sonst kein Farbstoff, kein Bodensatz; Kolben sehen im allgemeinen gleichmäßig aus.

Nach 4 Tagen: Ziemlich starke Trübung, Kolben 6 und 7 etwas stärker getrübt als 5, 8, 9; auf Kolben 6 und 7 dünne schwach rosenrote, nicht zusammenhängende Haut, auf den anderen Kolben schwache Ansätze zur Hautbildung, kein Farbstoff, kein Bodensatz; Kolben sehen gleichmäßig aus.

Nach 5 Tagen: Starke Trübung, auf allen Kolben nicht zusammenhängende Haut; auf Kolben 6 ist die Haut schwach rosenrot, auf den 
anderen Kolben farblos, kein Farbstoff, wenig Bodensatz; Kolben sehen gleichmäßig aus.

Nach 6 Tagen: Starke Trübung, auf Kolben 6 keine Haut, dafür am Boden viel Bakterienmasse, auf Kolben 7, 8, 9 farblose Haut, am Boden weniger Bakterienmasse, kein Farbstoff; Kolben sehen im allgemeinen gleichmäßig aus.

Nach 7 Tagen: Starke Trübung, auf Kolben 7 und 9 keine Haut, etwas Haut auf Kolben 8, kein Farbstoff, viel Bodensatz; Kolben sehen gleichmäßig aus.

Nach 8 Tagen: Sehr starke Trübung, auf Kolben 8 etwas Haut, welche zum Teil untergetaucht ist, auf Kolben 9 keine Haut; in beiden Kolben am Boden viel Bakterienmasse; die Haut scheint bald nach Bildung immer wieder zu Boden zu sinken; keine Farbstoffbildung; Kolben sehen gleichmäßig aus.

Nach 9 Tagen: Wie am Tage vorher, keine Haut, Bouillon gelb, viel Bodensatz; Kolben sehen gleichmäßig aus.

Nach 10 Tagen: Wie am Tage vorher.

Tabelle Nr. 38.

0,4602 g Ameisensäure als Natriumformiat bei $17^{\circ}$.

Bakterium: Bac. Plymouthensis Kràl.

\begin{tabular}{c|c|c|c|c|c|c}
\hline \hline $\begin{array}{c}\text { Zeit } \\
\text { in } \\
\text { Tagen }\end{array}$ & $\begin{array}{c}\text { Kolben } \\
\text { Nr. }\end{array}$ & Kalomel & $\begin{array}{c}\text { Noch } \\
\text { vorhandene } \\
\text { HCOOH } \\
\mathrm{g}\end{array}$ & $\begin{array}{c}\text { Noch } \\
\text { vorhandene } \\
\text { HCOOH } \\
\%\end{array}$ & $\begin{array}{c}\text { Vergoren } \\
\text { HCOOH }\end{array}$ & $\begin{array}{c}\text { Vergoren } \\
\text { HCOOH } \\
\text { g }\end{array}$ \\
\hline 1 & 1 & 4,5128 & 0,4410 & 95,83 & 0,0192 & 4,17 \\
2 & 3 & 4,0828 & 0,3990 & 86,70 & 0,0611 & 13,30 \\
3 & 5 & 3,9532 & 0,3863 & 83,95 & 0,0739 & 16,05 \\
4 & 7 & 3,9324 & 0,3843 & 83,50 & 0,0759 & 16,50 \\
5 & 9 & 3,9364 & 0,3847 & 83,59 & 0,0755 & 16,41
\end{tabular}

Tabelle Nr. 39.

$0,4602 \mathrm{~g}$ Ameisensäure als Natriumformiat bei $17^{\circ}$.

Bakterium: Bac. Plymouthensis Kràl.

\begin{tabular}{c|c|c|c|c|c|c}
\hline \hline $\begin{array}{c}\text { Zeit } \\
\text { in } \\
\text { Tagen }\end{array}$ & $\begin{array}{c}\text { Kolben } \\
\text { Nr. }\end{array}$ & Kalomel & $\begin{array}{c}\text { Noch } \\
\text { vorhandene } \\
\text { HCOOH } \\
\mathbf{g}\end{array}$ & $\begin{array}{c}\text { Noch } \\
\text { vorhandene } \\
\text { HCOOH } \\
\%\end{array}$ & $\begin{array}{c}\text { Vergoren } \\
\text { HCOOH } \\
\mathrm{g}\end{array}$ & $\begin{array}{c}\text { Vergoren } \\
\text { HCOOH } \\
\%\end{array}$ \\
\hline 1 & 2 & 4,5014 & 0,4399 & 95,59 & 0,0203 & 4,41 \\
2 & 4 & 4,0956 & 0,4002 & 86,97 & 0,0600 & 13,03 \\
3 & 6 & 3,9726 & 0,3882 & 84,36 & 0,0720 & 15,64 \\
4 & 8 & 3,9154 & 0,3826 & 83,14 & 0,0776 & 16,86 \\
5 & 10 & 3,8982 & 0,3810 & 82,78 & 0,0792 & 17,22
\end{tabular}


Die Kolben wurden nach der 2. Methode besät. Die Neutralisation der Bouillon wurde mit Hilfe von Phenolphthalein als Indikator durchgeführt.

Makroskopische Erscheinungen.

Nach 1 Tag: Mittelstarke Trübung, keine Haut, kein Farbstoff, kein Bodensatz, an der Oberfläche vereinzelte kleine Schaumblasen; Kolben sehen gleichmäßig aus.

Nach 2 Tagen: Trübung stärker als am Tage vorher, ganz kleine rosenrote Hautschollen, sonst kein Farbstoff, kein Bodensatz; Kolben sehen gleichmäßig aus.

Nach 3 Tagen: Starke Trübung, Kolben 5 etwas weniger stark gefärbt als 5, 9, 10, viele sehr kleine Hautschollen von rosenroter Farbe, sonst keine Farbstoffbildung, etwas Bodensatz, anscheinend zu Boden gesunkene Hautschollen; Kolben sehen gleichmäßig aus.

Nach 4 Tagen: Starke Trübung, Hautbildung nicht fortgeschritten, die ganze Flüssigkeit ist schwach rötlich gefärbt, etwas mehr Bodensatz als am Tage vorher; Kolben sehen gleichmäßig aus.

Nach 5 Tagen: Wie am Tage vorher; Kolben sehen gleichmäßig aus.

Versuchsreihe 37 wurde 6 Wochen früher als Versuchsreihe 38 und 39 , welche gleichzeitig angesetzt wurden, angesetzt.

Vergleich der gefundenen relativen Werte.

\begin{tabular}{|c|c|c|c|c|}
\hline \multicolumn{3}{|c|}{ Tabelle Nr. 37} & 38 & 39 \\
\hline & Tag & 4,44 & 4,17 & 4,41 \\
\hline 2. & , & 12,56 & 13,30 & 13,03 \\
\hline 3. & > & - & 16,05 & 15,64 \\
\hline 4. & > & 14,52 & 16,50 & 16,86 \\
\hline 5. & D & 23,52 & 16,41 & 17,22 \\
\hline 6. & D & 30,69 & - & - \\
\hline 7. & , & 34,50 & - & - \\
\hline 8. & , & 37,08 & - & - \\
\hline 9. & > & 38,51 & - & - \\
\hline 10. & , & 40,12 & - & - \\
\hline
\end{tabular}

Da die in Versuchsreihe 37 gefundenen Werte bedeutend von den in Versuchsreihe 38 und 39 gefundenen Werten abweichen, und da die Werte in 38 und 39 ganz gut übereinstimmen, soll nur das Mittel aus 38 und 39 genommen werden. 38 und 39 wurden zu gleicher Zeit angesetzt.

Mittlere Tabelle aus 38 und 39.

\begin{tabular}{|c|c|}
\hline 1. Tag & 4,29 \\
\hline 2. & 13,17 \\
\hline 3: \$ & 15,85 \\
\hline 4. $>$ & 16,68 \\
\hline 5. > & 16,82 \\
\hline
\end{tabular}


Abweichung der gefundenen von den mittleren Werten.

\begin{tabular}{|c|c|c|c|}
\hline Tabelle & Nr. 37 & 38 & 39 \\
\hline 1. Tag & $+0,15$ & $-0,12$ & $+0,12$ \\
\hline 2. $>$ & $-0,61$ & $+0,13$ & $-0,14$ \\
\hline 3. & - & $+0,20$ & $-0,21$ \\
\hline 4. $>$ & $-2,16$ & $-0,18$ & $+0,18$ \\
\hline 5. \ & $+6,70$ & $-0,41$ & $+0,40$ \\
\hline
\end{tabular}

Die Abweichungen der gefundenen von den mittleren Werten sind bei 38 und 39, abgesehen von den Zahlen des letzten Tages, recht klein, dagegen bei 37 recht groß.

Menge der während der einzelnen Tage vergorenen Ameisensäure.

Innerhalb sind vergoren $\mathrm{HCOOH}$

\begin{tabular}{|c|c|c|c|c|}
\hline & & Tabelle Nr. 37 & Mittel aus 38 & und 39 \\
\hline & ages & 4,44 & & 4,29 \\
\hline 2. & 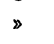 & 8,12 & & 8,88 \\
\hline 3. & $»$ & - & & 2,68 \\
\hline 4. & 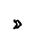 & - & & 0,83 \\
\hline 5. & $»$ & 9,00 & & 0,14 \\
\hline 6 . & $\triangleright$ & 7,17 & & \\
\hline 7. & $\triangleright$ & 3,81 & & \\
\hline 8. & $\triangleright$ & 2,58 & & \\
\hline 9. & $»$ & 1,43 & & \\
\hline 10. & $\gg$ & 1,61 & & \\
\hline
\end{tabular}

Vergleich der gefundenen absoluten Werte.

\begin{tabular}{|c|c|c|c|c|}
\hline & Tabelle & Nr. 37 & 38 & 39 \\
\hline & Tag & 204 & 192 & 203 \\
\hline 2. & , & 578 & 612 & 600 \\
\hline 3. & 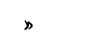 & - & 739 & 720 \\
\hline 4. & $D$ & 668 & 759 & 776 \\
\hline 5. & $\triangleright$ & 1082 & 755 & 792 \\
\hline 6. & $\triangleright$ & 1413 & & \\
\hline 7. & $\triangleright$ & 1587 & & \\
\hline 8. & $\nu$ & 1706 & & \\
\hline 9 . & 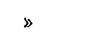 & 1772 & & \\
\hline & $\triangleright$ & 1846 & & \\
\hline
\end{tabular}

Mittlere Tabelle aus 38 und 39.
1. Tag
198
2. 》
606
$3 . \gg$
730
$4 .>$
$76 \dot{8}$
5. »
773 
Beiträge zur Biochemie der Mikroorganismen. III.

Abweichung der gefundenen von den mittleren Werten.

\begin{tabular}{lllll}
\multicolumn{2}{c}{ Tabelle Nr. 37} & 38 & 39 \\
\hline 1. Tag & +6 & -6 & +5 \\
2. & -28 & +6 & -6 \\
3. & - & +9 & -10 \\
4. & -100 & -9 & +8 \\
5. & +309 & -18 & +19
\end{tabular}

Menge der während der einzelnen Tage vergorenen

Ameisensäure.

\begin{tabular}{|c|c|c|}
\hline $\begin{array}{c}\text { Innerhalb } \\
\text { des }\end{array}$ & Tabelle Nr. 37 & $\begin{array}{l}\text { sind vergoren } \mathrm{HCOOH} \\
\text { Mittel aus } 38 \text { und } 39\end{array}$ \\
\hline 1. Tages & 204 & 198 \\
\hline 2. > & 374 & 408 \\
\hline 3. & - & 124 \\
\hline 4. & - & 38 \\
\hline 5. & 414 & \\
\hline 6. & 331 & \\
\hline 7. & 174 & \\
\hline 8. > & 119 & \\
\hline 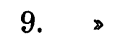 & 66 & \\
\hline 10. & 74 & \\
\hline
\end{tabular}

Versuchsreihe 38 und 39 zeigen untereinander, abgesehen von den Zahlen des letzten Tages, eine recht gute Übereinstimmung; beide Versuchsreihen wurden gleichzeitig mit derselben Bouillon angesetzt. Versuchsreihe 37 zeigt mit 38, 39 keine gute Übereinstimmung. Diese Nichtübereinstimmung drückt sich in den makroskopischen Erscheinungen aus. Versuchsreihe 37 zeigt auch sonstige Unregelmäßigkeiten, welche sich ebenfalls aus den makroskopischen Erscheinungen herauslesen lassen; dagegen zeigen 38 und 39 einen regelmäßigen Gärungsverlauf, und dieser drückt sich auch in den makroskopischen Erscheinungen aus. Die Unterschiede zwischen diesen beiden Gruppen von Versuchsreihen lassen sich wohl auch auf eine verschiedene Zusammensetzung der zur Anwendung gekommenen Bouillon zurückführen; die Abweichung am 5. Tage in Versuchsreihe 38, 39 wird wohl durch eine Verschiedenheit in der Luftzuführung bedingt sein. Für Vergleichszwecke soll nur das Mittel aus den Versuchsreihen 38 und 39 herangezogen werden.

In 5 Tagen werden $0,0773 \mathrm{~g}=16,82 \%$ Ameisensäure vergoren. Die größte Gärungsintensität liegt innerhalb des

Hoppe-Seyler's Zeitschrift f. physiol. Chemie. LXVII. 
2. Tages; innerhalb dieses Tages werden $0,0408 \mathrm{~g}=8,88 \%$ Ameisensäure vergoren; nach diesem Tage fällt die Gärungsintensität rasch ab und sie scheint nach 5 Tagen ihr Ende erreicht $\mathrm{zu}$ haben.

\section{2. $21^{\circ}$.}

Tabelle Nr. 40.

0,4602 g Ameisensäure als Natriumformiat bei $21^{\circ}$.

Bakterium: Bac. Plymouthensis Kràl.

\begin{tabular}{c|c|c|c|c|c|c}
\hline \hline $\begin{array}{c}\text { Zeit } \\
\text { in } \\
\text { Tagen }\end{array}$ & $\begin{array}{c}\text { Kolben } \\
\text { Nr. }\end{array}$ & $\begin{array}{c}\text { Kalomel } \\
\mathrm{g}\end{array}$ & $\begin{array}{c}\text { Noch } \\
\text { vorhandene } \\
\text { HCOOH. } \\
\mathrm{g}\end{array}$ & $\begin{array}{c}\text { Noch } \\
\text { vorhandene } \\
\text { HCOOH } \\
\%\end{array}$ & $\begin{array}{c}\text { Vergoren } \\
\text { HCOOH } \\
\mathrm{g}\end{array}$ & $\begin{array}{c}\text { Vergoren } \\
\text { HCOOH } \\
\%\end{array}$ \\
\hline 1 & 1 & 4,4662 & 0,4365 & 94,84 & 0,0237 & 5,16 \\
2 & 3 & 3,9872 & 0,3897 & 84,67 & 0,0705 & 15,33 \\
3 & 5 & 3,8030 & 0,3716 & 80,76 & 0,0886 & 19,24 \\
4 & 7 & 3,5180 & 0,3438 & 74,70 & 0,1164 & 25,30 \\
5 & 9 & 3,5058 & 0,3426 & 74,45 & 0,1176 & 25,55
\end{tabular}

Tabelle Nr. 41.

$0,4602 \mathrm{~g}$ Ameisensäure als Natriumformiat bei $21^{\circ}$.

Bakterium: Bac. Plymouthensis Kràl.

\begin{tabular}{c|c|c|c|c|c|c}
\hline $\begin{array}{c}\text { Zeit } \\
\text { in } \\
\text { Tagen }\end{array}$ & $\begin{array}{c}\text { Kolben } \\
\text { Nr. }\end{array}$ & $\begin{array}{c}\text { Kalomel } \\
\mathbf{g}\end{array}$ & $\begin{array}{c}\text { Noch } \\
\text { vorhandene } \\
\text { HCOOH } \\
\mathbf{g}\end{array}$ & $\begin{array}{c}\text { Noch } \\
\text { vorhandene } \\
\text { HCOOH } \\
\%\end{array}$ & $\begin{array}{c}\text { Vergoren } \\
\text { HCOOH } \\
\mathbf{g}\end{array}$ & $\begin{array}{c}\text { Vergoren } \\
\text { HCOOH } \\
\%\end{array}$ \\
\hline 1 & 2 & 4,4814 & 0,4380 & 95,17 & 0,0222 & 4,83 \\
2 & 4 & 4,0028 & 0,3912 & 85,00 & 0,0690 & 15,00 \\
3 & 6 & 3,7846 & 0,3699 & 80,37 & 0,0903 & 19,63 \\
4 & 8 & 3,5340 & 0,3455 & 75,05 & 0,1147 & 24,95 \\
5 & - & - & - & - & - & -
\end{tabular}

Die Kolben wurden nach der 2. Methode besät. Die Bouillon wurde mit Hilfe von Phenolphthalein als Indikator neutralisiert.

Makroskopische Erscheinungen.

Nach 1 Tag: Mittelstarke Trübung, keine Haut, kein Farbstoff, auf der Oberfläche einige kleine Schaumblasen, kein Bodensatz; Kolben sehen gleichmäßig aus.

Nach 2 Tagen: Sehr starke Trübung, ganz feine rosenrote Haut, sonst kein Farbstoff, kein Bodensatz; Kolben sehen gleichmäßig aus. 
Nach 3 Tagen: Sehr starke Trübung, keine Haut, kein Farbstoff, etwas Bodensatz; Kolben sehen gleichmäßig aus.

Nach \& Tagen: Sehr starke Trübung, feine farblose zusammenhängende Haut, kein Farbstoff, etwas Bodensatz; Kolben sehen gleichmäßig aus.

Nach 5 Tagen: Sehr starke Trübung, Haut zum größten Teil zu Boden gesunken, kein Farbstoff, viel Bodensatz; Kolben sehen gleichmäßig aus.

Versuchsreihe 40 und 41 wurden $\mathrm{zu}$ gleicher Zeit angesetzt.

Vergleich der gefundenen relativen Werte.

\begin{tabular}{|c|c|c|}
\hline & r. 40 & 41 \\
\hline 1. $\mathrm{Tag}$ & 5,16 & 4,83 \\
\hline 2. $>$ & 15,33 & 15,00 \\
\hline & 19,24 & 19,63 \\
\hline 4. & 25,30 & 24,95 \\
\hline 5. & 25,55 & - \\
\hline
\end{tabular}

Mittlere Tabelle.

\begin{tabular}{|c|c|}
\hline 1. $\mathrm{Tag}$ & 5,00 \\
\hline 2. & 15,17 \\
\hline 3. & 19,44 \\
\hline , & 25,13 \\
\hline 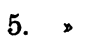 & 25,55 \\
\hline
\end{tabular}

Abweichung der gefundenen von den mittleren Werten.

\begin{tabular}{ccc} 
& Tabelle Nr. 40 & 41 \\
\hline 1. Tag & $+0,16$ & $-0,17$ \\
2. & $+0,16$ & $-0,17$ \\
$3 .:$ & $\pm 0,20$ & $+0,19$ \\
$4 .:$ & $+0,17$ & $-0,18$ \\
5. & $\pm 0,00$ & -
\end{tabular}

Die Abweichungen der gefundenen von den mittleren Werten sind recht klein; sie schwanken zwischen $+0,19 \%$ und $-0,20 \%$.

Menge der während der einzelnen Tage vergorenen Ameisensäure.

(Berechnet aus der mittleren Tabelle.) Innerhalb des
1. Tages
2.
5,00
3. $\gg$
10,17
$4 .>$
4,27
5. $>$
5,69
0,42

sind vergoren $\mathrm{HCOOH}$ 
Menge der während der einzelnen Tage vergorenen $\mathrm{HCOOH}$. Innerhalb sind vergoren $\mathrm{HCOOH}$

\begin{tabular}{lrr} 
des & Tabelle Nr. 40 & \multicolumn{1}{c}{41} \\
\hline 1. Tages & 5,16 & 4,83 \\
2. & 10,17 & 10,17 \\
3. & 3,91 & 4,63 \\
4. & 6,06 & 5,32 \\
5. & 0,25 & -
\end{tabular}

Vergleich der gefundenen absoluten Werte.

\begin{tabular}{|c|c|c|}
\hline & r. 40 & 41 \\
\hline 1. $\mathrm{Tag}$ & 237 & 222 \\
\hline 2. & 705 & 690 \\
\hline 3. & 886 & 903 \\
\hline 4. $>$ & 1164 & 1147 \\
\hline 5. , & 1176 & - \\
\hline
\end{tabular}

Mittlere Tabelle.
1. Tag
230
2.
698
3.
895
4. ,
1156
5. $\triangleright$
1176

Abweichung der gefundenen von den mittleren Werten.

\begin{tabular}{lrc}
\multicolumn{2}{c}{ Tabelle Nr. 40} & \multicolumn{1}{c}{41} \\
\hline 1. Tag & +7 & -8 \\
2. & +7 & -8 \\
3. & -9 & +8 \\
$4 .:$ & +8 & -9 \\
5. & \pm 0 & -
\end{tabular}

Die Abweichungen der gefundenen von den mittleren Werten sind recht klein, sie schwanken zwischen $+0,0008 \mathrm{~g}$ und $-0,0009 \mathrm{~g}$; halten sich also innerhalb der Versuchsfehler.

Menge der während der einzelnen Tage vergorenen Ameisensäure.

(Berechnet aus der mittleren Tabelle.) Innerhalb des

1. Tages sind vergoren $\mathrm{HCOOH}$

2., 230

3. ,

468

$4 .$,

197

5. ग 261

20 
Menge der während der einzelnen Tage vergorenen Ameisensäure.

Innerhalb sind vergoren $\mathrm{HCOOH}$

\begin{tabular}{|c|c|c|}
\hline des & Tabellen Nr. 40 & 41 \\
\hline 1. Tages & 237 & 222 \\
\hline 2. \ & 468 & 46 \\
\hline 3. & 181 & 21 \\
\hline 4. 》 & 278 & 2 \\
\hline 5. 2 & 12 & \\
\hline
\end{tabular}

Die beiden Versuchsreihen, welche mit derselben Bouillon angesetzt wurden, stimmen sehr gut überein, Abweichungen, welche auf eine Verschiedenartigkeit der Luftzuführung zurückgeführt werden müßten, kommen nicht vor.

Nach 5 Tagen sind $0,1176 \mathrm{~g}=25,55 \%$ Ameisensäure vergoren. Die größte Gärungsintensität liegt innerhalb des 2. Tages; innerhalb dieses Tages werden $0,0468 \mathrm{~g}=10,17 \%$ Ameisensäure vergoren. Die Gärungsintensität fällt dann allmählich ab und scheint nach 5 Tagen fastihr Ende erreicht zu haben.

\section{3. $27^{\circ}$.}

Ein Teil der Versuchsreihen, welche über die Vergärung der Ameisensäure durch Bac. Plymouthensis Kràl bei $27^{\circ}$ vorliegen, wurde im Sommersemester 1908 ausgeführt (Versuchsreihe 42, 43, 44, 45), ein anderer Teil im Wintersemester 1908/09 (Versuchsreihe 46, 47 und 48); zwischen der Ausführung dieser beiden Gruppen von Versuchsreihen liegt ein Zeitraum von $3^{1 / 2}-4$ Monaten. Da diese beiden Gruppen von Versuchsreihen keine gute Übereinstimmung zeigen, sollen sie besonders aufgeführt werden.

Tabelle Nr. 42.

$0,4602 \mathrm{~g}$ Ameisensäure als Natriumformiat bei $27^{\circ}$.

Bakterium: Bac. Plymouthensis Kràl.

\begin{tabular}{c|c|c|c|c|c}
\hline $\begin{array}{c}\text { Zeit } \\
\text { in } \\
\text { Tagen }\end{array}$ & Kalomel & $\begin{array}{c}\text { Noch } \\
\text { vorhandene } \\
\text { HCOOH } \\
\mathbf{g}\end{array}$ & $\begin{array}{c}\text { gorhandene } \\
\text { HCOOH } \\
\mathbf{0} / 0\end{array}$ & $\begin{array}{c}\text { Vergoren } \\
\text { HCOOH } \\
\mathbf{g}\end{array}$ & $\begin{array}{c}\text { Vergoren } \\
\text { HCOOH } \\
\%\end{array}$ \\
\hline 1 & 4,4310 & 0,4330 & 94,09 & 0,0272 & 5,91 \\
2 & 4,0428 & 0,3951 & 85,85 & 0,0651 & 14,15 \\
3 & 3,7196 & 0,3635 & 78,99 & 0,0967 & 21,01 \\
4 & 3,3698 & 0,3293 & 71,56 & 0,1309 & 28,44 \\
5 & - & - & - & - & -
\end{tabular}


Tabelle Nr. 43.

$0,4602 \mathrm{~g}$ Ameisensäure als Natriumformiat bei $27^{\circ}$.

Bakterium: Bac. Plymouthensis Kràl.

\begin{tabular}{c|c|c|c|c|c}
\hline \hline $\begin{array}{c}\text { Zeit } \\
\text { in } \\
\text { Tagen }\end{array}$ & $\begin{array}{c}\text { Kalomel } \\
\mathbf{g}\end{array}$ & $\begin{array}{c}\text { Noch } \\
\text { vorhandene } \\
\text { HCOOH } \\
\mathbf{g}\end{array}$ & $\begin{array}{c}\text { Noch } \\
\text { vorhandene } \\
\text { HCOOH } \\
\%\end{array}$ & $\begin{array}{c}\text { Vergoren } \\
\text { HCOOH } \\
\mathbf{g}\end{array}$ & $\begin{array}{c}\text { Vergoren } \\
\text { HCOOH } \\
\%\end{array}$ \\
\hline 1 & 4,5054 & 0,4403 & 95,67 & 0,0199 & 4,33 \\
2 & 3,9371 & 0,3848 & 83,61 & 0,0754 & 16,39 \\
3 & - & - & - & - & - \\
4 & 3,3996 & 0,3322 & 72,19 & 0,1280 & 27,81 \\
5 & 3,2654 & 0,3191 & 69,34 & 0,1411 & 30,66
\end{tabular}

Tabelle Nr. 44.

$0,4602 \mathrm{~g}$ Ameisensäure als Natriumformiat bei $27^{\circ}$.

Bakterium: Bac. Plymouthensis Kràl.

\begin{tabular}{c|c|c|c|c|c}
\hline \hline $\begin{array}{c}\text { Zeit } \\
\text { in } \\
\text { Tagen }\end{array}$ & $\begin{array}{c}\text { Kalomel } \\
\mathbf{g}\end{array}$ & $\begin{array}{c}\text { Noch } \\
\text { vorhandene } \\
\text { HCOOH } \\
\mathbf{g}\end{array}$ & $\begin{array}{c}\text { Noch } \\
\text { vorhandene } \\
\text { HCOOH } \\
\%\end{array}$ & $\begin{array}{c}\text { Vergoren } \\
\text { HCOOH } \\
\mathbf{g}\end{array}$ & $\begin{array}{c}\text { Vergoren } \\
\text { HCOOH } \\
\%\end{array}$ \\
\hline 1 & 4,5006 & 0,4398 & 95,57 & 0,0204 & 4,43 \\
2 & 3,9686 & 0,3878 & 84,28 & 0,0724 & 15,72 \\
3 & 3,7052 & 0,3621 & 78,68 & 0,0981 & 21,32 \\
4 & - & - & - & - & - \\
5 & 3,5428 & 0,3462 & 75,23 & 0,1140 & 24,77
\end{tabular}

Tabelle Nr. 45.

$0,4602 \mathrm{~g}$ Ameisensăure als Natriumformiat bei $27^{\circ}$.

Bakterium: Bac. Plymouthensis Kràl.

\begin{tabular}{|c|c|c|c|c|c|}
\hline $\begin{array}{c}\text { Zeit } \\
\text { in } \\
\text { Tagen }\end{array}$ & $\begin{array}{c}\text { Kalomel } \\
\mathbf{g}\end{array}$ & $\begin{array}{c}\text { Noch } \\
\text { vorhandene } \\
\text { HCOOH } \\
\mathbf{g}\end{array}$ & $\begin{array}{c}\text { Noch } \\
\text { vorhandene } \\
\text { HCOOH } \\
\%\end{array}$ & $\begin{array}{c}\text { Vergoren } \\
\text { HCOOH } \\
\text { g }\end{array}$ & $\begin{array}{c}\text { Vergoren } \\
\mathrm{HCOOH} \\
\%\end{array}$ \\
\hline 1 & - & - & - & - & - \\
\hline 2 & 3,9724 & 0,3882 & 84,35 & 0,0720 & 15,65 \\
\hline 3 & 3,7130 & 0,3629 & 78,85 & 0,0973 & 21,15 \\
\hline 4 & 3,5956 & 0,3514 & 76,35 & 0,1088 & 23,65 \\
\hline 5 & 3,5514 & 0,3471 & 75,42 & 0,1131 & 24,58 \\
\hline
\end{tabular}


Die Neutralisation der Bouillon erfolgte bei diesen Versuchsreihen mit Lackmuspapier als Indikator; die Kolben wurden nach Methode 1 geimpft.

Versuchsreihen 42, 43 wurden gleichzeitig angesetzt und ebenso Versuchsreihen 44, 45. Zwischen der Ausführung der beiden Versuchsreiben (42, 43 und 44, 45) lag ein Zwischenraum von 2 Wochen.

Vergleich der gefundenen relativen Werte.

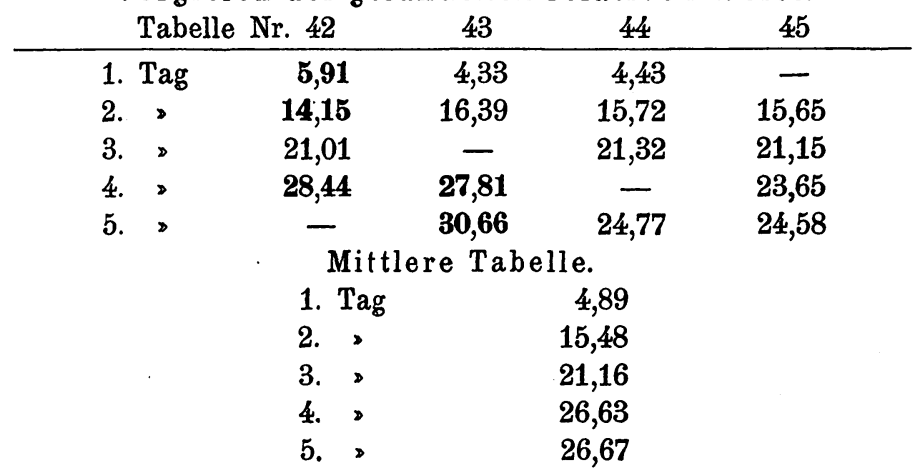

Abweichung der gefundenen von den mittleren Werten.

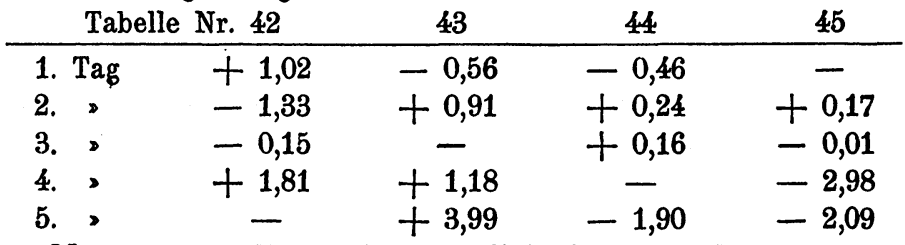

Merzt man die wahrscheinlich falschen Zahlen aus, so erhält man folgende korrigierte Tabelle.

$\begin{array}{rr}\text { Korrigierte mittlere Tabell } & 4,38 \\ \text { 1. Tag } & 15,92 \\ 2 . \searrow & 21,16 \\ 3 . \text {. } & 23,65 \\ 4 . \text {. } & 24,68\end{array}$

Abweichung der gefundenen von den mittleren Werten.

\begin{tabular}{lcccc}
\multicolumn{2}{c}{ Tabelle Nr. 42 } & 43 & 44 & 45 \\
\hline 1. Tag & $+1,53$ & $-0,05$ & $+0,05$ & - \\
2. & $-1,77$ & $+0,47$ & $-0,20$ & $-0,27$ \\
3. & $-0,15$ & - & $+0,16$ & $-0,01$ \\
4. & $+4,79$ & $+4,16$ & - & $\pm 0,00$ \\
5. & - & $+5,98$ & $+0,09$ & $-0,10$
\end{tabular}


Abgesehen von den eingerahmten Zahlen bewegen sich die Differenzen zwischen den Zahlen $+0,47 \%$ und $-0,27 \%$.

$\begin{array}{cr}\text { Innerhalb des } & \text { sind vergoren Ame } \\ \text { 1. Tages } & 4,38 \% \\ 2 . & 11,54 \% \\ 3 . & 5,24 \% \\ 4 . & 2,49 \% \\ 5 . & 1,03 \%\end{array}$

Vergleich der gefundenen absoluten Werte.

\begin{tabular}{|c|c|c|c|c|}
\hline Tabell & Nr. 42 & 43 & 44 & 45 \\
\hline 1. $\mathrm{Tag}$ & 272 & 199 & 204 & - \\
\hline 2. $>$ & 651 & 754 & 724 & 720 \\
\hline 3. & 967 & - & 981 & 973 \\
\hline 4. & 1309 & 1280 & - & 1088 \\
\hline 5. & - & 1411 & 1140 & 1131 \\
\hline
\end{tabular}

Mittlere Tabelle.

\begin{tabular}{|c|c|}
\hline 1. $\mathrm{Tag}$ & 225 \\
\hline 2. D & 712 \\
\hline 3. > & 974 \\
\hline $4 .>$ & 1226 \\
\hline 5. & 1227 \\
\hline
\end{tabular}

Abweichung der gefundenen von den mittleren Werten.

\begin{tabular}{lccccc}
\multicolumn{2}{c}{ Tabelle Nr. 42} & 43 & 44 & 45 \\
\hline 1. Tag & +47 & -26 & -21 & - \\
2. & -61 & +42 & +12 & +8 \\
3. & -7 & - & +7 & -1 \\
$4 .:$ & +83 & +54 & - & -138 \\
5. $:$ & - & +184 & -87 & -96
\end{tabular}

Merzt man die wahrscheinlich fehlerhaften Zahlen aus, so erhält man folgende korrigierte mittlere Tabelle:

$\begin{array}{rr}\text { Korrigierte mittlere } & \text { Tab } \\ \text { 1. Tag } & 202 \\ \text { 2. } & 733 \\ \text { 3. } & 974 \\ \text { 4. } & 1088 \\ \text { 5. } & 1136\end{array}$


Abweichung der gefundenen von den korrigierten mittleren Werten.

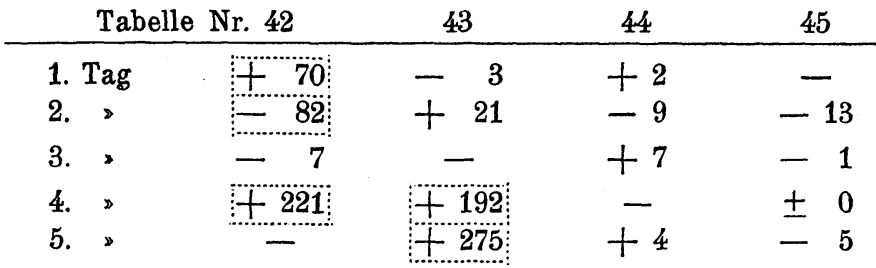

Abgesehen von den eingerahmten Zahlen bewegen sich die Differenzen zwischen $+0,0021 \mathrm{~g}$ und $-0,0013 \mathrm{~g}$.

$\begin{array}{rr}\text { Innerhalb des } & \text { sind vergoren A } \\ \text { 1. Tages } & 202 \\ 2 . \text { : } & 531 \\ 3 . & 241 \\ 4 . & 114 \\ 5 . & \end{array}$

Tabelle Nr. 46.

$0,4602 \mathrm{~g}$ Ameisensäure als Natriumformiat bei $27^{\circ}$. Bakterium: Bac. Plymouthensis Kràl.

\begin{tabular}{c|c|c|c|c|c|c}
\hline \hline $\begin{array}{c}\text { Zeit } \\
\text { in } \\
\text { Tagen }\end{array}$ & $\begin{array}{c}\text { Kolben } \\
\text { Nr. }\end{array}$ & $\begin{array}{c}\text { Kalomel } \\
\mathbf{g}\end{array}$ & $\begin{array}{c}\text { Noch } \\
\text { vorhandene } \\
\text { HCOOH } \\
\mathbf{g}\end{array}$ & $\begin{array}{c}\text { Noch } \\
\text { vorhandene } \\
\text { HCOOH } \\
\%\end{array}$ & $\begin{array}{c}\text { Vergoren } \\
\text { HCOOH } \\
\mathbf{g}\end{array}$ & $\begin{array}{c}\text { Vergoren } \\
\text { HCOOH } \\
\%\end{array}$ \\
\hline 1 & 1 & 4,5238 & 0,4421 & 96,06 & 0,0181 & 3,94 \\
2 & 2 & 4,0312 & 0,3939 & 85,60 & 0,0663 & 14,40 \\
3 & - & - & - & - & - & - \\
4 & 3 & 3,7546 & 0,3669 & 79,73 & 0,0933 & 20,27 \\
5 & 4 & 3,5828 & 0,3501 & 76,08 & 0,1101 & 23,92 \\
6 & 5 & 3,3652 & 0,3289 & 71,46 & 0,1313 & 28,54 \\
7 & 6 & 3,2438 & 0,3170 & 68,88 & 0,1432 & 31,12 \\
8 & 7 & 3,1336 & 0,3062 & 66,54 & 0,1540 & 33,46 \\
9 & 8 & 3,0382 & 0,2969 & 64,52 & 0,1633 & 35,48 \\
10 & 9 & 3,0051 & 0,2937 & 63,81 & 0,1665 & 36,19 \\
11 & 10 & 2,9934 & 0,2925 & 63,57 & 0,1677 & 36,43
\end{tabular}

Die Kolben wurden nach der 1. Methode geimpft. Die Neutralisation der Bouillon wurde mit Hilfe von Phenolphthalein als Indikator durchgeführt. 
Makroskopische Erscheinungen.

Nach 1 Tag: Ziemlich starke Trübung, keine Haut, nur an der Wandung des Glases, dort wo die Flüssigkeit aufhört, dünner, farbloser Ring von Bakterienhaut, kein Farbstoff, kein Bodensatz; Kolben sehen gleichmäßig aus.

Nach 2 Tagen: Sehr starke Trübung, schwache farblose zusammenhängende Haut, kein Farbstoff, kein Bodensatz, unangenehmer Geruch; Kolben sehen gleichmäßig aus.

Nach 3 Tagen: Sehr starke Trübung, schwache farblose zusammenhängende Haut, kein Farbstoff, kein Bodensatz, unangenehmer Geruch; Kolben sehen gleichmäßig aus.

Nach $\&$ Tagen: Sehr starke Trübung, keine Haut, kein Farbstoff, viel Bodensatz, unangenehmer Geruch; Kolben sehen gleichmäßig aus.

Nach 5 Tagen: Sehr starke Trübung, keine Haut, kein Farbstoff, viel Bodensatz, unangenehmer Geruch; Kolben sehen gleichmäßig aus.

Nach 6 Tagen: Sehr starke Trübung, keine Haut, kein roter Farbstoff, die Flüssigkeit ist gelb gefärbt, gelber als die ursprüngliche Bouillon, viel Bodensatz, unangenehmer Geruch; Kolben sehen gleichmäßig aus.

Nach 7 Tagen: Starke Trübung, keine Haut, kein roter Farbstoff, Flüssigkeit gelb, viel Bodensatz; Kolben sehen gleichmäßig aus.

Nach 8 Tagen: Sehr starke Trübung, keine Haut, kein roter Farbstoff, Bouillon gelb, viel Bodensatz; Kolben sehen gleichmäßig aus.

Nach 9 Tagen: Sehr starke Trübung, keine Haut, Flüssigkeit gelb mit einem Stich ins Rötliche, viel Bodensatz; Kolben sehen gleichmäßig aus.

Nach 10 Tagen: Wie am Tage vorher, die Flüssigkeit hat noch einen Stich mehr ins Rötliche; Kolben sehen gleichmäßig aus.

Tabelle Nr. 47.

$0,4602 \mathrm{~g}$ Ameisensäure als Natriumformiat bei $27^{\circ}$.

Bakterium: Bac. Plymouthensis Kràl.

\begin{tabular}{c|c|c|c|c|c|c}
\hline $\begin{array}{c}\text { Zeit } \\
\text { in } \\
\text { Tagen }\end{array}$ & $\begin{array}{c}\text { Kolben } \\
\text { Nr. }\end{array}$ & Kalomel & $\begin{array}{c}\text { Noch } \\
\text { vorhandene } \\
\text { HCOOH } \\
\mathbf{g}\end{array}$ & $\begin{array}{c}\text { Noch } \\
\text { vorhandene } \\
\text { HCOOH } \\
\%\end{array}$ & $\begin{array}{c}\text { Vergoren } \\
\text { HCOOH } \\
\mathbf{g}\end{array}$ & $\begin{array}{c}\text { Vergoren } \\
\text { HCOOH } \\
\%\end{array}$ \\
\hline 1 & 1 & 4,4944 & 0,4390 & 95,44 & 0,0212 & 4,56 \\
2 & 3 & 3,8168 & 0,3730 & 81,05 & 0,0872 & 18,95 \\
3 & 5 & 3,4662 & 0,3387 & 73,61 & 0,1215 & 26,39 \\
4 & 7 & 3,3648 & 0,3288 & 71,45 & 0,1314 & 28,55 \\
5 & 9 & 3,2226 & 0,3149 & 68,43 & 0,1453 & 31,57
\end{tabular}


Beiträge zur Biochemie der Mikroorganismen. III.

Tabelle Nr. 48.

$0,4602 \mathrm{~g}$ Ameisensäure als Natriumformiat bei $27^{\circ}$.

Bakterium: Bac. Plymouthensis Kràl.

\begin{tabular}{c|c|c|c|c|c|c}
\hline $\begin{array}{c}\text { Zeit } \\
\text { in } \\
\text { Tagen }\end{array}$ & $\begin{array}{c}\text { Kolben } \\
\text { Nr. }\end{array}$ & Kalomel & $\begin{array}{c}\text { Noch } \\
\text { vorhandene } \\
\text { HCOOH } \\
\mathbf{g}\end{array}$ & $\begin{array}{c}\text { Noch } \\
\text { vorhandene } \\
\text { HCOOH } \\
\%\end{array}$ & $\begin{array}{c}\text { Vergoren } \\
\text { HCOOH } \\
\mathrm{g}\end{array}$ & $\begin{array}{c}\text { Vergoren } \\
\text { HCOOH } \\
\%\end{array}$ \\
\hline 1 & 2 & 4,3844 & 0,4285 & 93,10 & 0,0317 & 6,90 \\
2 & 4 & 3,7716 & 0,3686 & 80,09 & 0,0916 & 19,91 \\
3 & 6 & 3,4944 & 0,3415 & 74,21 & 0,1187 & 25,79 \\
4 & 8 & 3,3488 & 0,3273 & 71,11 & 0,1329 & 28,89 \\
5 & 10 & 3,2766 & 0,3202 & 69,58 & 0,1400 & 30,42
\end{tabular}

Die Kolben wurden nach der zweiten Methode geimpft. Die Bouillon wurde mit Hilfe von Phenolphthalein als Indikator durchgeführt.

\section{Makroskopische Erscheinungen.}

Nach 1 Tag: Starke Trübung, keine Haut, kein Farbstoff, keine Schaumblasen, kein Bodensatz; Kolben sehen gleichmäßig aus.

Nach 2 Tagen: Sehr starke Trübung, sehr feine farblose Haut, kein Farbstoff, kein Bodensatz; Kolben sehen gleichmäßig aus.

Nach 3 Tagen: Sehr starke Trübung, feine, nicht zusammenhängende Haut, kein Farbstoff, etwas Bodensatz, unangenehmer Geruch; Kolben sehen gleichmäßig aus.

Nach 4 Tagen: Sehr starke Trübung, keine Haut, kein Farbstoff, ziemlich viel Bodensatz; Kolben sehen gleichmäßig aus. mäßig aus.

Nach 5 Tagen: Wie am Tage vorher; Kolben sehen gleich-

Vergleich der gefundenen relativen Werte.

\begin{tabular}{|c|c|c|c|c|}
\hline \multicolumn{3}{|c|}{ Tabelle Nr. 46} & 47 & 48 \\
\hline & Tag & 3,94 & 4,56 & 6,90 \\
\hline 2. & $>$ & 14,40 & 18,95 & 19,91 \\
\hline 3. & , & - & 26,39 & 25,79 \\
\hline 4. & > & 20,27 & 28,55 & 28,89 \\
\hline 5 . & $\triangleright$ & 23,92 & 31,57 & 30,42 \\
\hline 6 & , & 28,54 & - & - \\
\hline 7. & > & 31,12 & - & - \\
\hline 8 & $>$ & 33,46 & 一 & - \\
\hline 9 & $=$ & 35,48 & 一 & - \\
\hline 10 & $>$ & 36,19 & - & 一 \\
\hline & $\gg$ & 36,43 & - & - \\
\hline
\end{tabular}

Wegen der schlechten Übereinstimmung der verschiedenen 
Versuchsreihen ist es unzweckmäßig, eine mittlere Tabelle aufzustellen.

Menge der während der einzelnen Tage vergorenen Ameisensäure.

\begin{tabular}{|c|c|c|c|}
\hline Innerhalb & \multirow[b]{2}{*}{ Tabelle } & \multicolumn{2}{|c|}{ sind vergoren $\mathrm{HCOOH}$} \\
\hline des & & 47 & 48 \\
\hline 1. Tages & 3,94 & 4,56 & 6,90 \\
\hline 2. & 10,46 & 14,39 & 13,01 \\
\hline 3. & - & 7,44 & 5,88 \\
\hline 4. & - & 2,16 & 3,10 \\
\hline 5. & 3,65 & 3,02 & 1,53 \\
\hline 6. & 4,62 & - & - \\
\hline 7. & 2,58 & - & - \\
\hline 8. & 2,34 & - & - \\
\hline 9. & 2,02 & - & - \\
\hline 10. 》 & 0,71 & - & - \\
\hline 11. & 0,24 & - & - \\
\hline
\end{tabular}

Vergleich der gefundenen absoluten Werte.

\begin{tabular}{|c|c|c|c|c|}
\hline \multicolumn{2}{|c|}{ Tabell } & Nr. 46 & 47 & 48 \\
\hline 1. & Tag & 181 & 212 & 317 \\
\hline 2. & 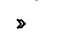 & 663 & 872 & 916 \\
\hline 3. & $\triangleright$ & - & 1215 & 1187 \\
\hline 4. & $\triangleright$ & 933 & 1314 & 1329 \\
\hline 5. & 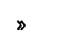 & 1101 & 1453 & 1400 \\
\hline 6. & 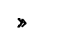 & 1313 & - & - \\
\hline 7. & D & 1432 & - & - \\
\hline 8. & $\gg$ & 1540 & - & - \\
\hline 9. & $»$ & 1633 & - & - \\
\hline 10. & D & 1665 & - & - \\
\hline 11. & , & 1667 & - & - \\
\hline
\end{tabular}

Menge der während der einzelnen Tage vergorenen Ameisensäure.

\begin{tabular}{|c|c|c|c|}
\hline \multirow{2}{*}{$\begin{array}{l}\text { Innerhalb } \\
\text { des }\end{array}$} & \multirow[b]{2}{*}{ Tabelle Nr. 46} & \multicolumn{2}{|c|}{ sind vergoren $\mathrm{HCOOH}$} \\
\hline & & 47 & 48 \\
\hline 1. Tages & 181 & 212 & 317 \\
\hline $2 . \quad \gg$ & 482 & 660 & 599 \\
\hline 3. & - & 343 & 271 \\
\hline 4. & - & 99 & 142 \\
\hline o. & 168 & 139 & 71 \\
\hline 6. & 212 & - & - \\
\hline 7. & 119 & - & - \\
\hline 8. & 108 & - & - \\
\hline 9. & 93 & - & - \\
\hline 10. & 32 & - & - \\
\hline 11. & 2 & - & - \\
\hline
\end{tabular}


Die Versuchsreihen 42, 43, 44, 45 zeigen im allgemeinen keine so gute Übereinstimmung wie die analogen Versuchsreihen $(10,11,12,13)$ bei Bac. Prodigiosus Kràl. Aber die Übereinstimmung ist doch wiederum so gut, daß man auch hier schließen kann, daß es wohl möglich ist, bei verschiedenen Darstellungen eine Nährbouillon zu erhalten, welche den Bakterien analoge Lebensbedingungen bietet. Die Abweichungen, die innerhalb dieser 4 Versuchsreihen vorkommen, lassen sich wohl auf eine Verschiedenartigkeit des Luftwechsels zurückführen. - Für Vergleichszwecke soll die korrigierte mittlere Tabelle verwendet werden.

Nach 5 Tagen sind $0,1136 \mathrm{~g}=24,68 \%$ Ameisensäure vergoren. Die größte Gärungsintensität liegt innerhalb des 2. Tages; innerhalb dieses Tages werden $0,0531 \mathrm{~g}=11,54 \%$ Ameisensäure vergoren. Nach diesem Tage fällt die Gärungsintensität $a b$, und sie scheint nach 5 Tagen so ziemlich ihr Ende erreicht zu haben.

Die Versuchsreihen 46, 47, 48 zeigen untereinander keine gute Übereinstimmung; auch die Versuchsreihen 47 und 48 zeigen keine so gute Übereinstimmung, wie man sie wohl erwarten dürfte, da für beide Versuchsreihen dieselbe Bouillon verwendet wurde. Die Unterschiede zwischen Versuchsreihen 46 einerseits und 47, 48 anderseits lassen sich wohl auf eine Verschiedenartigkeit der Bouillon zurückführen, aber für die Unterschiede zwischen den beiden Versuchsreihen 47 und 48 läßt sich nur eine Verschiedenartigkeit des Luftwechsels verantwortlich machen.

Nach 5 Tagen sind vergoren Ameisensäure:

$$
\begin{aligned}
& \text { Tab. } 460,1101 \mathrm{~g}=23,92 \% \\
& \text { » } 470,1453 »=31,57 \% \\
& \text { » } 480,1400 »=30,42 \%
\end{aligned}
$$

Die größte Gärungsintensität liegt, wie aus Versuçhsreihe 47 und 48 hervorgeht, innerhalb des 2 . Tages. Innerhalb dieses Tages werden vergoren Ameisensäure:

$$
\begin{gathered}
\text { Tab. } 470,0660 \mathrm{~g}=14,39 \% \\
» 48 \quad 0,0599 *=14,01 \%
\end{gathered}
$$


Nach diesem Tage fällt die Gärungsintensität allmählich $a b$, hat aber nach 5 Tagen noch nicht ihr Ende erreicht.

Zum Vergleich der beiden Gruppen von Versuchsreihen wird es wohl am besten sein, die erhaltenen Zahlen noch einmal nebeneinander zu legen. Von der Gruppe 42, 43, 44, 45 soll hierzu nicht das Mittel aus den 4 Versuchsreihen, sondern jede einzelne Versuchsreihe verwendet werden.

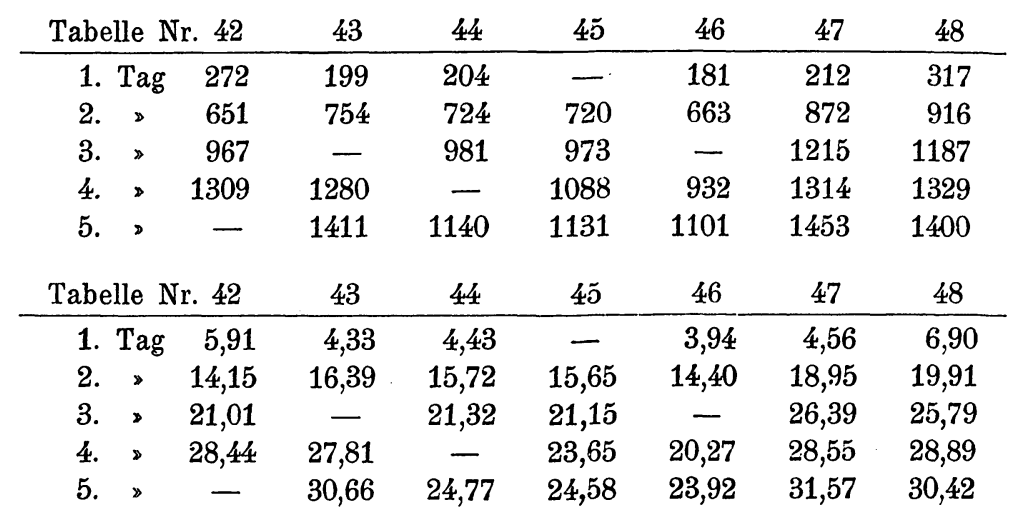

Betrachtet man zunächst die beiden Gruppen von Versuchsreihen, so wird man finden, daß die Differenzen im allgemeinen recht große sind; aber bei genauerem Vergleich wird man auch sehen, daß manche Zahlen der ersten Gruppe mit denen der zweiten Gruppe eine recht gute Übereinstimmung zeigen. Hieraus geht wohl hervor, daß nicht eine physiologische Veränderung der Bakterien die Ursache der Verschiedenheit ist, sondern andere Ursachen, nämlich Verschiedenheit der Bouillon oder ungleichmäßiger Luftzutritt. An eine Veränderung des physiologischen Zustandes der Bakterien wäre immerhin zu denken, da zwischen der Ausführung der ersten Gruppe von Versuchsreihen und der zweiten ein Zeitraum von $3^{1 / 2}$ bis 4 Monaten liegt. Die Annahme, daß eine Veränderung des physiologischen Zustandes der Bakterien die Ursache der Differenzen sei, wird auch noch dadurch unwahrscheinlich, daß die Differenzen zwischen der ersten Gruppe von Versuchsreihen und Versuchsreihe 46, zwischen welchen ein Zeitraum von $3^{1 / 2}-4$ Monaten liegt, kleiner sind als die zwischen Versuchs- 
reihe 46 und Versuchsreihe 47 und 48 , zwischen welchen ein Zeitraum von 6 Wochen liegt.

Nehmen wir nun aus den sämtlichen Zahlen das Mittel, so sind nach 5 Tagen $0,1273 \mathrm{~g}=27,65 \%$ Ameisensäure vergoren. Die größte Gärungsintensität liegt innerhalb des 2. Tages; an diesem Tage werden $0,0526 \mathrm{~g}=11,30 \%$ Ameisensäure vergoren.

\section{4. $37^{\circ}$.}

Tabelle Nr. 49.

$0,4602 \mathrm{~g}$ Ameisensäure als Natriumformiat bei $37^{\circ}$.

Bakterium: Bac. Plymouthensis Kràl.

\begin{tabular}{c|c|c|c|c|c}
\hline $\begin{array}{c}\text { Zeit } \\
\text { in } \\
\text { Tagen }\end{array}$ & Kalomel & $\begin{array}{c}\text { Noch } \\
\text { vorhandene } \\
\text { HCOOH } \\
\mathbf{g}\end{array}$ & $\begin{array}{c}\text { Noch } \\
\text { vorhandene } \\
\text { HCOOH } \\
\%\end{array}$ & $\begin{array}{c}\text { Vergoren } \\
\text { HCOOH } \\
\mathbf{g}\end{array}$ & $\begin{array}{c}\text { Vergoren } \\
\text { HCOOH } \\
\text { \% }\end{array}$ \\
\hline 1 & 4,7046 & 0,4598 & 99,90 & 0,0004 & 0,10 \\
2 & $\mathbf{4 , 7 1 2 0}$ & 0,4605 & 100,06 & 0,0003 & 0,06 \\
3 & 4,7114 & 0,4604 & 100,05 & 0,0002 & 0,05 \\
4 & 4,7078 & 0,4601 & 99,97 & 0,0001 & 0,03 \\
5 & - & - & - & - & -
\end{tabular}

Tabelle Nr. 50.

0,4602 g Ameisensäure als Natriumformiat bei $37^{\circ}$.

Bakterium: Bac. Plymouthensis Kràl.

\begin{tabular}{|c|c|c|c|c|c|}
\hline $\begin{array}{c}\text { Zeit } \\
\text { in } \\
\text { Tagen }\end{array}$ & $\begin{array}{c}\text { Kalomel } \\
\text { g }\end{array}$ & $\begin{array}{c}\text { Noch } \\
\text { vorhandene } \\
\text { HCOOH } \\
\text { g }\end{array}$ & $\begin{array}{c}\text { Noch } \\
\text { vorhandene } \\
\text { HCOOH } \\
\% \%\end{array}$ & $\begin{array}{c}\text { Vergoren } \\
\mathrm{HCOOH} \\
\mathbf{g}\end{array}$ & $\begin{array}{c}\text { Vergoren } \\
\text { HCOOH } \\
\%\end{array}$ \\
\hline 1 & 4,7094 & 0,4602 & 100,01 & 0,0000 & 0,01 \\
\hline 2 & 4,7092 & 0,4602 & 100,00 & 0,0000 & 0,00 \\
\hline 3 & - & - & - & - & - \\
\hline 4 & 4,6966 & 0,4590 & 99,73 & 0,0012 & 0,27 \\
\hline
\end{tabular}


Tabelle Nr. 51.

0,4602 g Ameisensäure als Natriumformiat bei $37^{\circ}$.

Bakterium: Bac.'Plymouthensis Kràl.

\begin{tabular}{c|c|c|c|c|c}
\hline $\begin{array}{c}\text { Zeit } \\
\text { in } \\
\text { Tagen }\end{array}$ & $\mathrm{g}$ & $\begin{array}{c}\text { Kalomel } \\
\text { vorhandene } \\
\mathrm{g}\end{array}$ & $\begin{array}{c}\text { NoOH } \\
\text { vorhandene } \\
\text { HCOOH } \\
\%\end{array}$ & $\begin{array}{c}\text { Vergoren } \\
\text { HCOOH } \\
\mathrm{g}\end{array}$ & $\begin{array}{c}\text { Vergoren } \\
\text { HCOOH } \\
\%\end{array}$ \\
\hline 1 & 4,7086 & 0,4602 & 99,99 & 0,0000 & 0,01 \\
2 & 4,7086 & 0,4600 & 99,95 & 0,0002 & 0,05 \\
3 & 4,7072 & 0,4600 & 99,96 & 0,0002 & 0,04 \\
4 & 4,7032 & 0,4596 & 99,87 & 0,0006 & 0,13 \\
5 & 4,7092 & 0,4602 & 100,00 & 0,0000 & 0,0
\end{tabular}

Tabelle Nr. 52.

0,4602 g Ameisensäure als Natriumformiat bei $37^{\circ}$.

Bakterium: Bac. Plymouthensis Kràl.

\begin{tabular}{c|c|c|c|c|c}
\hline \hline $\begin{array}{c}\text { Zeit } \\
\text { in } \\
\text { Tagen }\end{array}$ & Kalomel & $\begin{array}{c}\text { Noch } \\
\text { vorhandene } \\
\text { HCOOH } \\
\mathbf{g}\end{array}$ & $\begin{array}{c}\text { Noch } \\
\text { vorhandene } \\
\text { HCOOH } \\
\%\end{array}$ & $\begin{array}{c}\text { Vergoren } \\
\text { HCOOH } \\
\mathbf{g}\end{array}$ & $\begin{array}{c}\text { Vergoren } \\
\text { HCOOH } \\
\%\end{array}$ \\
\hline 1 & 4,7122 & 0,4605 & 100,07 & 0,0003 & 0,07 \\
2 & 4,7118 & 0,4605 & 100,06 & 0,0003 & 0,06 \\
3 & 4,6996 & 0,4593 & 99,80 & 0,0009 & 0,20 \\
4 & 4,7010 & 0,4594 & 99,83 & 0,0008 & 0,17 \\
5 & - & - & - & - & -
\end{tabular}

Die Neutralisation der Nährbouillon wurde mit Hilfe von Lackmuspapier als Indikator durchgeführt; die Kolben wurden nach Methode 1 geimpft.

Vergleich der bei den verschiedenen Temperaturen erhaltenen Zahlen.

Hier fällt zunächst die Tatsache in die Augen, daß bei $37^{\circ}$ keine Ameisensäure mehr vergoren wird, diese Temperatur braucht also fernerhin nicht mehr berücksichtigt zu werden. Die größte Gärungsintensität liegt bei allen Temperaturen innerhalb des 2. Tages.

An diesem Tage werden vergoren

bei $17^{\circ} 0,0408 \mathrm{~g}=8,88 \%$ Ameisensäure

» $21^{\circ} 0,0468 \mathrm{~g}=10,17 \%$ »

» $27^{\circ} 0,0526 \mathrm{~g}=11,30 \%$ » 
Die Gärung hat bei $17^{\circ}$ und $21^{\circ}$ nach 5 Tagen fast ihr Ende erreicht, bei $27^{\circ}$ aber noch nicht.

Nach 5 Tagen sind vergoren

bei $17^{\circ} 0,0773 \mathrm{~g}=16,82 \%$ Ameisensäure

» $21^{\circ} 0,1176 \mathrm{~g}=25,55 \%$ *

» $27^{\circ} 0,1273 \mathrm{~g}=27,65 \%$ »

Die Zahlen sagen aus, daß innerhalb 5 Tagen bei $21^{\circ}$ mehr Ameisensäure vergoren wird als bei $17^{\circ}$, und bei $27^{\circ}$ mehr Ameisensäure als bei $21^{\circ}$. Die Differenz zwischen $17^{\circ}$ und $21^{\circ}$ beträgt $0,0403 \mathrm{~g}=8,73 \%$ und die zwischen $21^{\circ}$ und $27^{\circ} 0,0097 \mathrm{~g}=2,10^{\circ} \%$. Der Sprung von $17^{\circ}$ auf $21^{\circ}$ (Unterschied $4^{\circ}$ ) ist bedeutend größer, annähernd $4 \mathrm{mal}$, als der zwischen $21^{\circ}$ und $27^{\circ}$ (Unterschied $6^{\circ}$ ).

Vergleich der mit Calciumformiat und mit Natriumformiat erhaltenen Zahlen.

Während mit Natriumformiat bei $37^{\circ}$ überhaupt keine Vergärung mehr erhalten wird, wird Calciumformiat bei dieser Temperatur noch vergoren. Innerhalb 5 Tagen werden $0,0383 \mathrm{~g}$ $=8,32 \%$ Ameisensäure vergoren. Bei $27^{\circ}$ werden beide Salze vergoren. Bei Anwendung von Natriumformiat wird innerhalb 5 Tagen $0,1273 \mathrm{~g}=27,65 \%$, bei Anwendung von Calciumformiat dagegen nur $0,406 \mathrm{~g}=8,61 \%$ Ameisensäure vergoren.

\section{B. Stamm K. G.A.}

Tabelle Nr. 53.

0,4602 g Ameisensăure als Natriumformiat bei $17^{\circ}$.

Bakterium: Bac. Plymouthensis K. G. A.

\begin{tabular}{c|c|c|c|c|c|c}
\hline $\begin{array}{c}\text { Zeit } \\
\text { in } \\
\text { Tagen }\end{array}$ & $\begin{array}{c}\text { Kolben } \\
\mathrm{Nr} .\end{array}$ & $\mathrm{Kalomel}$ & $\begin{array}{c}\text { Noch } \\
\text { vorhandene } \\
\text { HCOOH } \\
\mathrm{g}\end{array}$ & $\begin{array}{c}\text { Noch } \\
\text { vorhandene } \\
\text { HCOOH } \\
\%\end{array}$ & $\begin{array}{c}\text { Vergoren } \\
\text { HCOOH } \\
\mathrm{g}\end{array}$ & $\begin{array}{c}\text { Vergoren } \\
\text { HCOOH } \\
\%\end{array}$ \\
\hline $\mathbf{1}$ & $\mathbf{1}$ & $\mathbf{4 , 5 8 9 2}$ & 0,4485 & 97,45 & 0,0117 & 2,55 \\
$\mathbf{2}$ & $\mathbf{3}$ & $\mathbf{4 , 4 2 4 6}$ & 0,4324 & 93,96 & 0,0278 & 6,04 \\
$\mathbf{3}$ & $\mathbf{5}$ & 4,3186 & 0,4220 & 91,71 & 0,0382 & $\mathbf{8 , 2 9}$ \\
$\mathbf{4}$ & $\mathbf{7}$ & $\mathbf{4 , 1 2 5 6}$ & 0,4032 & 87,61 & 0,0570 & 12,39 \\
$\mathbf{5}$ & $\mathbf{9}$ & 3,9224 & 0,3833 & 83,29 & 0,0769 & 16,71
\end{tabular}

Hoppe-Seyler's Zeitschrift f. physiol. Chemie. LXVII. 
Tabelle Nr. 54.

$0,4602 \mathrm{~g}$ Ameisensäure als Natriumformiat bei $17^{\circ}$.

Bakterium: Bac. Plymouthensis K. G. A.

\begin{tabular}{c|c|c|c|c|c|c}
\hline \hline $\begin{array}{c}\text { Zeit } \\
\text { in } \\
\text { Tagen }\end{array}$ & $\begin{array}{c}\text { Kolben } \\
\text { Nr. }\end{array}$ & $\begin{array}{c}\text { Kalomel } \\
\mathbf{g}\end{array}$ & $\begin{array}{c}\text { Noch } \\
\text { vorhandene } \\
\text { HCOOH } \\
\mathbf{g}\end{array}$ & $\begin{array}{c}\text { Noch } \\
\text { vorhandene } \\
\text { HCOOH } \\
\%\end{array}$ & $\begin{array}{c}\text { Vergoren } \\
\text { HCOOH } \\
\mathbf{g}\end{array}$ & $\begin{array}{c}\text { Vergoren } \\
\text { HCOOH } \\
\%\end{array}$ \\
\hline 1 & 2 & 4,6104 & 0,4506 & 97,90 & 0,0096 & 2,10 \\
2 & 4 & 4,4036 & 0,4304 & 93,51 & 0,0298 & 6,49 \\
3 & 6 & 4,3198 & 0,4222 & 91,73 & 0,0380 & 8,27 \\
4 & 8 & 4,1338 & 0,4040 & 87,78 & 0,0562 & 12,22 \\
5 & 10 & 3,9416 & 0,3852 & 83,70 & 0,0750 & 16,30
\end{tabular}

Die Neutralisation der Nährbouillon wurde mit Hilfe von Phenolphthalein als Indikator durchgeführt; die Kolben wurden nach Methode 2 geimpft.

\section{Makroskopische Erscheinungen.}

Nach 1 Tag: Schwache Trübung, keine Haut, kein Farbstoff, kein Bodensatz; Kolben sehen gleichmäßig aus.

Nach 2 Tagen: Trübung etwas stärker als am Tage vorher, an der Wandung des Glases, dort wo die Flüssigkeit auf hört, schwach rosenroter Ring, auf der Oberfläche einige kleine rosenrote Hautschollen, die sehr leicht bei geringer Erschütterung zu Boden sinken, Flüssigkeit nicht gefärbt, etwas Bodensatz; Kolben sehen gleichmäßig aus.

Nach 3 Tagen: Trübung kaum stärker als am Tage vorher, die Hautschollen sind etwas größer geworden und haben sich intensiver gefärbt, auch die Farbe des roten Ringes ist intensiver geworden, die Flüssigkeit ist nicht gefärbt, etwas mehr Bodensatz als am Tage vorher; Kolben sehen gleichmäßig aus.

Nach 4 Tagen: Trübung nicht stärker als am Tage vorher; die Hautschollen sind etwas größer geworden und hellrot gefärbt, ziemlich viel Bodensatz; Kolben sehen gleichmäßig aus.

Nach 5 Tagen: Wie am Tage vorher; Kolben sehen gleichmäßig aus. 
Beiträge zur Biochemie der Mikroorganismen. III.

Tabelle Nr. 55.

$0,4602 \mathrm{~g}$ Ameisensäure als Natriumformiat bei $17^{\circ}$.

Bakterium: Bac. Plymouthensis K. G. A.

\begin{tabular}{c|c|c|c|c|c|c}
\hline \hline $\begin{array}{c}\text { Zeit } \\
\text { in } \\
\text { Tagen }\end{array}$ & $\begin{array}{c}\text { Kolben } \\
\text { Nr. }\end{array}$ & Kalomel & $\begin{array}{c}\text { Noch } \\
\text { vorhandene } \\
\text { HCOOH } \\
\mathrm{g}\end{array}$ & $\begin{array}{c}\text { Noch } \\
\text { vorhandene } \\
\text { HCOOH } \\
\%\end{array}$ & $\begin{array}{c}\text { Vergoren } \\
\text { HCOOH } \\
\mathbf{g}\end{array}$ & $\begin{array}{c}\text { Vergoren } \\
\text { HCOOH } \\
\%\end{array}$ \\
\hline 1 & 1 & 4,5806 & 0,4476 & $\ldots 7,27$ & 0,0126 & 2,73 \\
2 & 3 & 4,4306 & 0,4330 & 94,09 & 0,0272 & 5,91 \\
3 & 5 & 4,3154 & 0,4217 & 91,64 & 0,0385 & 8,36 \\
4 & 7 & 4,2368 & 0,4140 & 89,97 & 0,0462 & 10,03 \\
5 & 9 & 4,1902 & 0,4095 & 88,98 & 0,0507 & 11,02
\end{tabular}

Tabelle Nr. 56.

$0,4602 \mathrm{~g}$ Ameisensäure als Natriumformiat bei $17^{\circ}$.

Bakterium: Bac. Plymouthensis K. G. A.

\begin{tabular}{c|c|c|c|c|c|c}
\hline $\begin{array}{c}\text { Zeit } \\
\text { in } \\
\text { Tagen }\end{array}$ & $\begin{array}{c}\text { Kolben } \\
\mathrm{Nr} .\end{array}$ & $\begin{array}{c}\text { Kalomel } \\
\mathrm{g}\end{array}$ & $\begin{array}{c}\text { Noch } \\
\text { vorhandene } \\
\mathrm{HCOOH} \\
\mathrm{g}\end{array}$ & $\begin{array}{c}\text { Noch } \\
\text { vorhandene } \\
\text { HCOOH } \\
\%\end{array}$ & $\begin{array}{c}\text { Vergoren } \\
\text { HCOOH } \\
\mathrm{g}\end{array}$ & $\begin{array}{c}\text { Vergoren } \\
\text { HCOOH } \\
\%\end{array}$ \\
\hline 1 & 2 & 4,5982 & 0,4494 & 97,65 & 0,0108 & 2,35 \\
2 & 4 & 4,4182 & 0,4318 & 93,82 & 0,0284 & 6,18 \\
3 & 6 & 4,3112 & 0,4213 & 91,55 & 0,0389 & 8,45 \\
4 & 8 & 4,2446 & 0,4148 & 90,14 & 0,0454 & 9,86 \\
5 & 10 & 4,1828 & 0,4088 & 88,82 & 0,0514 & 11,18
\end{tabular}

Die Neutralisation der Nährbouillon wurde mit Hilfe von Phenolphthalein als Indikator durchgeführt; die Kolben,wurden nach Methode 2 geimpft.

Makroskopische Erscheinungen.

Nach 1 Tag: Schwache Trübung, keine Haut, kein Farbstoff, etwas Bodensatz; Kolben sehen gleichmäßig aus.

Nach 2 Tagen: Schwache Trübung, auf der Oberfläche einige kleine rosenrote Hautschollen, an der Wandung des Glases, dort wo die Flüssigkeit aufhört, schwach rosenroter Ring, sonst kein Farbstoff, etwas Bodensatz; Kolben sehen gleichmäßig aus.

$\mathrm{Nach} 3$ Tagen: Mittelstarke Trübung, viele kleine rosenrote Hautschollen, der rosenrote Ring ist intensiver gefärbt als am Tage vorher, ziemlich viel Bodensatz; Kolben sehen gleichmäßig aus. 
$\mathrm{Nach} \&$ Tagen: Starke Trübung, viele kleine isolierte Hautschollen, die Färbung der Hautschollen ist intensiver geworden als am Tage vorher, sonst kein Farbstoff, ziemlich viel Bodensatz; Kolben sehen gleichmäßig aus. mäßig aus.

Nach 5 Tagen: Wie am Tage vorher; Kolben sehen gleich-

Versuchsreihen 55,56 , welche gleichzeitig angestellt wurden, wurden 3 Wochen später als Versuchsreihen 53, 54, welche ebenfalls gleichzeitig angestellt wurden, durchgeführt.

Vergleich der gefundenen relativen Werten.

\begin{tabular}{lrrrr} 
Tabelle & Nr. 53 & \multicolumn{1}{c}{54} & \multicolumn{1}{c}{55} & \multicolumn{1}{c}{56} \\
\hline 1. Tag & 2,55 & 2,10 & 2,73 & 2,53 \\
2. & 6,04 & 6,49 & 5,91 & 6,18 \\
3. \ & 8,28 & 8,27 & 8,36 & 8,45 \\
$4 .>$ & 12,39 & 12,22 & 10,03 & 9,86 \\
5. & 16,71 & 16,30 & 11,02 & 11,18
\end{tabular}

Beim Vergleich der beiden Parallelversuche 53, 54 und 55 , 56, die mit einem Zeitunterschied von 3 Wochen ausgeführt wurden, findet man, daß die ersten 3 Tage gut übereinstimmende Werte geben. Bei den beiden letzten Tagen treten recht bedeutende Verschiedenheiten hervor; während die zu derselben Zeit angestellten Versuchsreihen untereinander auch hier gute Übereinstimmung zeigen ( 53 und $54-55$ und 56 ), wird bei den Versuchsreihen 53, 54 bedeutend mehr Ameisensäure vergoren, als bei den Versuchsreihen 5ら̆, 56. Für diese Differenzen kommt als Ursache der physiologische Zustand der Bakterien und die ungleiche Menge der Einsaat nicht in Betracht, denn dann hätten sich die Differenzen schon früher zeigen müssen; es bleibt nichts anderes übrig, als entweder die Verschiedenheit der Zusammensetzung der Nährbouillon oder der Verschiedenartigkeit des Luftzutrittes die Schuld zuzuschieben.

Mittlere Tabelle.

$\begin{array}{lr}\text { 1. Tag } & 2,48 \\ 2 . \searrow & 6,16 \\ 3 . \searrow & 8,34 \\ 4 . \searrow & 11,13 \\ 5 .> & 13,80\end{array}$


Abweichung der gefundenen von den mittleren Werten.

\begin{tabular}{ccccc}
\multicolumn{2}{c}{ Tabelle Nr. 53} & 54 & 55 & 56 \\
\hline 1. Tag & $+0,07$ & $-0,38$ & $+0,25$ & $+0,05$ \\
$2 .:$ & $-0,12$ & $+0,33$ & $-0,25$ & $+0,02$ \\
$3 .:$ & $-0,05$ & $-0,07$ & $+0,02$ & $+0,11$ \\
$4 .:$ & $+1,46$ & $+1,09$ & $-1,10$ & $-1,27$ \\
5. & $+2,91$ & $+2,50$ & $-2,78$ & $-2,62$
\end{tabular}

Die Abweichungen der gefundenen von den mittleren Werten sind in den ersten 3 Tagen recht klein, sie schwanken zwischen $+0,33 \%$ und $-0,38 \%$. Die Abweichungen der beiden Tage sind, weil die beiden Parallelversuchsreihen an diesen Tagen nicht übereinstimmen, natürlich recht groß. Da die gefundenen Werte der gleichzeitig angesetzten Versuchsreihen untereinander gut übereinstimmen, ist es nicht erlaubt, einer der beiden Parallelversuchsreihen den Vorzug zu geben und die andere nicht zu berücksichtigen. Eine korrigierte mittlere Tabelle läßt sich deshalb nicht berechnen. Für Vergleichszwecke dürfte es in diesem Fall am besten sein, aus beiden gleichzeitig angestellten Versuchsreihen das Mittel zu ziehen.

Mittlere Tabelle aus 53 und 54.

\begin{tabular}{|c|c|}
\hline 1. Tag & 2,33 \\
\hline 2. , & 6,27 \\
\hline 3. > & 8,28 \\
\hline 4. & 12,30 \\
\hline 5. > & 16,50 \\
\hline
\end{tabular}

Mittlere Tabelle aus 55 und 56.

\begin{tabular}{|c|c|}
\hline 1. Tag & 2,63 \\
\hline 2. , & 6,05 \\
\hline 3. & 8,41 \\
\hline 4. & 9,95 \\
\hline 5. & 11,10 \\
\hline
\end{tabular}

Menge der während der einzelnen Tage vergorenen Ameisensäure.

(Berechnet aus der mittleren Tabelle 53 und 54.)

Innerhalb des

1. Tages

2.,

3.

$4 . \$$

5. , sind vergoren $\mathrm{HCOOH}$

2,33

3,94

2,01

4,02

4,20 
Menge der während der einzelnen Tage vergorenen Ameisensäure.

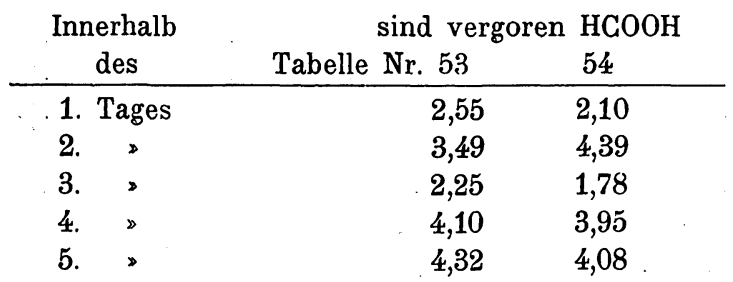

Menge der während der einzelnen Tage vergorenen Ameisensäure.

(Berechnet aus der mittleren Tabelle 55 und 56.) Innerhalb des
1. Tages
2. $\gg$
2,63
3. »
3,42
4. D
2,36
5.
1,54
1,15

sind vergoren $\mathrm{HCOOH}$

Vergleich der gefundenen absoluten Werte.

\begin{tabular}{|c|c|c|c|c|}
\hline Tahelle & Nr. 53 & 54 & 55 & 56 \\
\hline 1. Tag & 117 & 96 & 126 & 108 \\
\hline 2. & 278 & 298 & 272 & 284 \\
\hline 3. & 382 & 380 & 385 & 389 \\
\hline 4. $>$ & 570 & 562 & 462 & 454 \\
\hline 5. & 769 & 750 & 507 & 514 \\
\hline
\end{tabular}

Für den Vergleich der absoluten Werte gilt natürlich dasselbe, was beim Vergleich der relativen Werte gesagt worden ist. Mittlere Tabelle.
1. Tag
112
2. $\gg$
283
3. 》
384
4. $>$ 512
5.,
635

Die Abweichungen der gefundenen von den mittleren Werten sind in den ersten 3 Tagen recht klein, erreichen aber am 4. und 5. Tage eine recht beträchtliche Größe.

Mittlere Tabelle aus 53 und 54 .
1. Tag
107
2. \
288
3.
381
4. 》
566
5. 》
760 
Mittlere Tabelle aus 55 und 56.

$\begin{array}{ll}\text { 1. Tag } & 117 \\ 2 . \gg & 278 \\ 3 . \text { \. } & 387 \\ 4 . \gg & 458 \\ 5 .> & 512\end{array}$

Menge der während der einzelnen Tage vergorenen Ameisensäure.

(Berechnet aus der mittleren Tabelle von 53. und 54.) Innerhalb des
1. Tages
2. $\triangle$
107
3. "
181
$4 . \quad$
93
5.
185
194

sind vergoren $\mathrm{HCOOH}$

Menge der während der einzelnen Tage vergorenen Ameisensäure.

\begin{tabular}{crr}
$\begin{array}{c}\text { Innerhalb } \\
\text { des }\end{array}$ & \multicolumn{2}{c}{ sind vergoren $\mathrm{HCOOH}$} \\
\hline 1. Tages & Tabelle Nr. 53 & \multicolumn{1}{c}{54} \\
2. & 117 & 96 \\
3. & 161 & 202 \\
4. & 104 & 82 \\
5. & 188 & 182 \\
\end{tabular}

Menge der während der einzelnen Tage vergorenen Ameisensäure.

(Berechnet aus der mittleren Tabelle von 55 und 56.)

Innerhalb des

1. Tages

2.

3. ,

$4 . \quad$

5. sind vergoren $\mathrm{HCOOH}$

117

161

109

- 71

Die Ursache der Nichtübereinstimmung der beiden Gruppen von Versuchsreihen ist schon weiter oben diskutiert worden. In 5 .Tagen werden $0,0635 \mathrm{~g}=13,80 \%$ Ameisensäure vergoren. Die größte Gärungsintensität liegt innerhalb des 2. Tages; innerhalb dieses Tages werden $0,0171 \mathrm{~g}=3,68 \%$ Ameisensäure vergoren. Nach diesem Tage fällt die Gärungsintensität allmählich $a b$, ist aber nach 5 Tagen noch nicht beendet. 
Auffallend ist hier auch noch, daß trotzdem die makroskopischen Erscheinungen eine recht gute Übereinstimmung zeigen, doch in den erhaltenen Zahlen an den letzten beiden Tagen keine Übereinstimmung vorhanden ist.

\section{2. $21^{\circ}$.}

Tabelle Nr. 57.

$0,4602 \mathrm{~g}$ Ameisensäure als Natriumformiat bei $21^{\circ}$.

Bakterium: Bac. Plymouthensis K. G. A.

\begin{tabular}{c|c|c|c|c|c|c}
\hline $\begin{array}{c}\text { Zeit } \\
\text { in } \\
\text { Tagen }\end{array}$ & $\begin{array}{c}\text { Kolben } \\
\text { Nr. }\end{array}$ & Kalomel & $\begin{array}{c}\text { Noch } \\
\text { vorhandene } \\
\text { HCOOH } \\
\mathbf{g}\end{array}$ & $\begin{array}{c}\text { Noch } \\
\text { vorhandene } \\
\text { HCOOH } \\
\% / 0\end{array}$ & $\begin{array}{c}\text { Vergoren } \\
\text { HCOOH } \\
\mathbf{g}\end{array}$ & $\begin{array}{c}\text { Vergoren } \\
\text { HCOOH } \\
\%\end{array}$ \\
\hline 1 & 1 & 4,5342 & 0,4431 & 96,29 & 0,0171 & 3,71 \\
2 & 3 & 4,3256 & 0,4227 & 91,86 & 0,0375 & 8,14 \\
3 & 5 & 4,0956 & 0,4002 & 86,97 & 0,0600 & 13,03 \\
4 & 7 & 3,8432 & 0,3756 & 81,61 & 0,0846 & 18,39 \\
5 & 9 & 3,8220 & 0,3735 & 81,16 & 0,0867 & 18,84
\end{tabular}

Tabelle Nr. 58.

0,4602 g Ameisensäure als Natriumformiat bei $21^{\circ}$.

Bakterium: Bac. Plymouthensis K. G. A.

\begin{tabular}{c|c|c|c|c|c|c}
\hline \hline $\begin{array}{c}\text { Zeit } \\
\text { in } \\
\text { Tagen }\end{array}$ & $\begin{array}{c}\text { Kolben } \\
\text { Nr. }\end{array}$ & $\begin{array}{c}\text { Kalomel } \\
\mathbf{g}\end{array}$ & $\begin{array}{c}\text { Noch } \\
\text { vorhandene } \\
\text { HCOOH } \\
\mathbf{g}\end{array}$ & $\begin{array}{c}\text { Noch } \\
\text { vorhandene } \\
\text { HCOOH } \\
\text { \% }\end{array}$ & $\begin{array}{c}\text { Vergoren } \\
\text { HCOOH } \\
\mathbf{g}\end{array}$ & $\begin{array}{c}\text { Vergoren } \\
\text { HCOOH } \\
\%\end{array}$ \\
\hline 1 & 2 & 4,5438 & 0,4441 & 96,49 & 0,0161 & 3,51 \\
2 & 4 & 4,3280 & 0,4230 & 91,91 & 0,0372 & 8,09 \\
3 & 6 & 4,1992 & 9,4104 & 89,17 & 0,0498 & 10,83 \\
4 & 8 & 3,8586 & 0,3771 & 81,94 & 0,0831 & 18,06
\end{tabular}

Die Neutralisation der Nährbouillon wurde mit Hilfe von Phenolphthalein als Indikator durchgeführt; die Kolben wurden nach Methode 2 geimpft.

Makroskopische Erscheinungen.

Nach 1 Tag: Mittelstarke Trübung, keine Haut, kein Farbstof́, kein Bodensatz; Kolben sehen gleichmäßig aus.

Nach 2 Tagen: Trübung etwas stärker als am Tage vorher, arf 
der Oberfläche einige kleine isolierte Hautschollen, kein Farbstoff, wenig Bodensatz; Kolben sehen gleichmäßig aus.

Nach 3 Tagen: Starke Trübung, auf der Oberfläche viele kleine isolierte Hautschollen, an der Wandung des Glases dort, wo die Flüssigkeit aufhört, schwach rosenrot gefärbter Ring, sonst kein Farbstoff, etwas Bodensatz; Kolben sehen gleichmäßig aus.

Nach 4 Tagen: Starke Trübung, auf der Oberfläche ziemlich viel rosa gefärbte Hautschollen, sonst kein Farbstoff, wenig Bodensatz; Kolben sehen gleichmäßig aus.

Nach 5 Tagen: Wie am Tage vorher.

Tabelle Nr. 59.

$0,4602 \mathrm{~g}$ Ameisensäure als Natriumformiat bei $21^{\circ}$.

Bakterium: Bac. Plymouthensis K. G. A.

\begin{tabular}{c|c|c|c|c|c|c}
\hline \hline $\begin{array}{c}\text { Zeit } \\
\text { in } \\
\text { Tagen }\end{array}$ & $\begin{array}{c}\text { Kolben } \\
\text { Nr. }\end{array}$ & Kalomel & $\begin{array}{c}\text { Noch } \\
\text { vorhandene } \\
\text { HCOOH } \\
\mathbf{g}\end{array}$ & $\begin{array}{c}\text { Noch } \\
\text { vorhandene } \\
\text { HCOOH } \\
\%\end{array}$ & $\begin{array}{c}\text { Vergoren } \\
\text { HCOOH } \\
\mathbf{g}\end{array}$ & $\begin{array}{c}\text { Vergoren } \\
\text { HCOOH } \\
\%\end{array}$ \\
\hline 1 & 1 & 4,5412 & 0,4438 & 96,43 & 0,0164 & 3,57 \\
2 & 3 & 4,2654 & 0,4168 & 90,58 & 0,0434 & 9,42 \\
3 & 5 & 4,0812 & 0,3988 & 86,67 & 0,0614 & 13,33 \\
4 & 7 & 3,9920 & 0,3901 & 84,77 & 0,0701 & 15,23 \\
5 & 9 & 3,9388 & 0,3849 & 83,64 & 0,0753 & 16,36
\end{tabular}

Tabelle Nr. 60.

0,4602 g Ameisensäure als Natriumformiat bei $21^{\circ}$.

Bakterium: Bac. Plymouthensis K. G. A.

\begin{tabular}{c|c|c|c|c|c|c}
\hline $\begin{array}{c}\text { Zeit } \\
\text { in } \\
\text { Tagen }\end{array}$ & $\begin{array}{c}\text { Kolben } \\
\mathrm{Nr}\end{array}$ & $\mathrm{Kalomel}$ & $\begin{array}{c}\text { Noch } \\
\text { vorhandene } \\
\text { HCOOH } \\
\mathrm{g}\end{array}$ & $\begin{array}{c}\text { 年orhandene } \\
\text { HCOOH } \\
\%\end{array}$ & $\begin{array}{c}\text { Vergoren } \\
\text { HCOOH } \\
\mathrm{g}\end{array}$ & $\begin{array}{c}\text { Vergoren } \\
\text { HCOOH } \\
\%\end{array}$ \\
\hline 1 & 2 & 4,5308 & 0,4428 & 96,21 & 0,0174 & 3,79 \\
2 & 4 & 4,2716 & 0,4174 & 90,71 & 0,0428 & 9,29 \\
3 & 6 & 4,0786 & 0,3986 & 86,61 & 0,0616 & 13,39 \\
4 & 8 & 3,9858 & 0,3895 & 84,64 & 0,0707 & 15,36 \\
5 & - & - & - & - & - & -
\end{tabular}

Die Neutralisation der Nährbouillon wurde mit Hilfe von Phenolphthalein als Indikator durchgeführt; die Kolben wurden nach Methode 2 geimpft. 
Makroskopische Erscheinungen.

Nach 1 Tag: Mittelstarke Trübung, keine Haut, kein Farbstoff, wenig Bodensatz; Kolben sehen gleichmäßig aus.

Nach 2 Tagen: Ziemlich starke Trübung, auf der Oberfläche einzelne kleine isolierte Hautschollen, kein Farbstoff, etwas Bodensatz; Kolben sehen gleichmäßig aus.

Nach 3 Tagen: Ziemlich starke Trübung, auf der Oberfläche einzelne rosenrote Hautschollen, sonst kein Farbstoff, etwas Bodensatz; Kolben sehen gleichmäßig aus.

Nach 4 Tagen: Starke Trübung, auf der Oberfläche einzelne rosenrote Hautschollen, sonst kein Farbstoff, etwas Bodensatz; Kolben sehen gleichmäßig aus.

Nach $\tilde{5}$ Tagen: Wie am Tage vorher.

Versuchsreihen 59, 60, welche zu gleicher Zeit angestellt wurden, wurden 3 Wochen später als Versuchsreihen 57, 58, welche ebenfalls gleichzeitig angestellt wurden, durchgeführt.

Vergleich der gefundenen relativen Werte.

\begin{tabular}{rrrrr}
\multicolumn{2}{c}{ Tabelle Nr. 57} & \multicolumn{1}{c}{58} & \multicolumn{1}{c}{59} & \multicolumn{1}{c}{60} \\
\hline 1. Tag & 3,71 & 3,51 & 3,57 & 3,79 \\
2. & 8,14 & 8,09 & 9,42 & 9,29 \\
$3 .>$ & 13,03 & 10,83 & 13,33 & 13,39 \\
4. > & 18,03 & 18,06 & 15,23 & 15,36 \\
5. > & 18,84 & - & 16,36 & -
\end{tabular}

Vergleicht man zunächst die beiden $z u$ gleicher Zeit angesetzten Versuchsreihen 57 und 58, so findet man, daß die Werte im allgemeinen gut übereinstimmen, nur der Wert 10,83 am 3. Tage fällt aus der Versuchsreihe beraus. Beim Vergleich der Menge der während der einzelnen Tage vergorenen Ameisensäure wird man finden, daß der Parallelwert 13,03 in diesem Falle der wahrscheinlichere ist; der Wert 10,83 wäre also bei der Berechnung der mittleren Tabelle auszuschalten. Die anderen beiden Versuchsreihen 59 und 60, welche ebenfalls $\mathrm{zu}$ gleicher Zeit angesetzt wurden, zeigen untereinander gute Übereinstimmung. Vergleicht man aber die beiden Versuchsreihen 57, 58 mit 59, 60, welche mit einem Zeitunterschied von 3 Wochen angesetzt wurden, so sind die Differenzen recht bedeutend. Am 2. Tage ist bei 59, $60 \mathrm{mehr}$ Ameisensäure vergoren als bei 57, 58. Am 3. Tage sind die Werte wieder annähernd dieselben, wenn von der Zahl 10,83, welche aus 
den weiter oben angeführten Gründen ausgeschieden werden soll, abgesehen wird. Am 4. Tage ist das Verhältnis umgekehrt wie am 2. Tage; hier ist bei 57,58 bedeutend mehr Ameisensäure vergoren als bei 59,60 und ebenso liegen die Verhältnisse am 5. Tage.

Die Ursache dieser Verschiedenheit dürfte wohl wieder in einer ungleichartigen Bouillon und in der Verschiedenartigkeit des Luftzutrittes zu suchen sein.

Mittlere Tabelle aus 57 und 58.
1. Tag
3,61
2. $\gg$
8,12
3.
13,03
4.
18,05
5.
18,84

Mittlere Tabelle aus 59 und 60.
1. Tag
3,68
2.,
9,36
3.
13,36
4. ,
15,30
5. $>$
16,36

Menge der während der einzelnen Tage vergorenen Ameisensäure.

(Berechnet aus der mittleren Tabelle 57 und 58.)

$\begin{array}{cr}\text { Innerhalb des } & \text { sind vergoren } \\ \text { 1. Tages } & 3,61 \\ \text { 2. } & 4,51 \\ \text { 3. } & 4,91 \\ \text { 4. } & 5,02 \\ \text { 5. } & 0,79\end{array}$

Menge der während der einzelnen Tage vergorenen Ameisensäure.

(Berechnet aus der mittleren Tabelle 59 und 60.)

$\begin{array}{cr}\text { Innerhalb des } & \text { sind vergoren } \\ \text { 1. Tages } & 3,68 \\ 2 . & 5,68 \\ 3 . & 4,00 \\ 4 . & 1,94 \\ 5 . & 1,06\end{array}$


Vergleich der gefundenen absoluten Werte.

\begin{tabular}{|c|c|c|c|c|}
\hline Tabell & Nr. 57 & 58 & 59 & 60 \\
\hline 1. Tag & 171 & 161 & 164 & 174 \\
\hline & 375 & 372 & 434 & 428 \\
\hline & 600 & 498 & 614 & 616 \\
\hline 4. & 846 & 831 & 701 & 707 \\
\hline 5. & 867 & - & 753 & - \\
\hline
\end{tabular}

Für diese Tabelle gilt natürlich dasselbe, was für den Vergleich der gefundenen relativen Werte gesagt worden ist. Mittlere Tabelle aus 57 und 58.
1. Tag
166
2.
374
3.
600
$4 . \gg$
839
5. $>$
867

Mittlere Tabelle aus 59 und 60 .
1. Tag
169
2. \
431
3. $>$ 615
4. $>$ 704
5. $>$
753

Menge der während der einzelnen Tage vergorenen Ameisensäure.

(Berechnet aus der mittleren Tabelle 57 und 58.) Innerhalb des sind vergoren $\mathrm{HCOOH}$
1. Tages
166
2.
208
3. $\gg$
226
4. $\triangleright$
239
$5 . \quad \triangleright$
28

Menge der während der einzelnen Tage vergorenen Ameisensäure.

(Berechnet aus der mittleren Tabelle 59 und 60.) Innerhalb des sind vergoren $\mathrm{HCOOH}$
1. Tages
169
2. $\gg$
262
3. $\triangleright$
184
$4 . \$$
99
$5 . \quad>$
49

Die Ursache der Verschiedenheit der beiden Versuchsreihen ist schon weiter oben diskutiert worden. 
Nach 5 Tagen werden im Mittel $0,0810 \mathrm{~g}=17,60 \%$ Ameisensäure vergoren. Die Gärungsintensität ist innerhalb des 2. Tages am größten; innerhalb dieses Tages werden im Mittel vergoren $0,0237 \mathrm{~g}=5,09 \%$ Ameisensäure.

Merkwürdig ist auch wieder hier, daß trotz der guten Übereinstimmung der makroskopischen Erscheinungen die erhaltenen Werte derartige Unterschiede zeigen.

\section{3. $27^{\circ}$.}

Tabelle Nr. 61.

0,4602 g Ameisensäure als Natriumformiat bei $27^{\circ}$.

Bakterium: Bac. Plymouthensis K.G.A.

\begin{tabular}{c|c|c|c|c|c|c}
\hline $\begin{array}{c}\text { Zeit } \\
\text { in } \\
\text { Tagen }\end{array}$ & $\begin{array}{c}\text { Kolben } \\
\text { Nr. }\end{array}$ & Kalomel & $\begin{array}{c}\text { Noch } \\
\text { vorhandene } \\
\text { HCOOH } \\
\mathrm{g}\end{array}$ & $\begin{array}{c}\text { Noch } \\
\text { vorhandene } \\
\text { HCOOH } \\
\%\end{array}$ & $\begin{array}{c}\text { Vergoren } \\
\text { HCOOH } \\
\mathrm{g}\end{array}$ & $\begin{array}{c}\text { Vergoren } \\
\text { HCOOH } \\
\%\end{array}$ \\
\hline $\mathbf{1}$ & $\mathbf{1}$ & $\mathbf{4 , 4 6 8 6}$ & 0,4367 & 94,89 & 0,0235 & 5,11 \\
$\mathbf{2}$ & $\mathbf{3}$ & $\mathbf{4 , 1 9 6 4}$ & 0,4101 & 89,11 & 0,0501 & 10,89 \\
$\mathbf{3}$ & $\mathbf{5}$ & 3,9574 & 0,3867 & 84,04 & 0,0735 & 15,96 \\
$\mathbf{4}$ & $\mathbf{7}$ & 3,7994 & 0,3713 & 80,68 & 0,0889 & 19,32 \\
$\mathbf{5}$ & $\mathbf{9}$ & 3,4256 & 0,3348 & 72,75 & 0,1254 & 27,25
\end{tabular}

Tabelle Nr. 62.

0,4602 g Ameisensäure als Natriumformiat bei $27^{\circ}$.

Bakterium: Bac. Plymouthensis K.G.A.

\begin{tabular}{c|c|c|c|c|c|c}
\hline $\begin{array}{c}\text { Zeit } \\
\text { in } \\
\text { Tagen }\end{array}$ & $\begin{array}{c}\text { Kolben } \\
\text { Nr. }\end{array}$ & $\begin{array}{c}\text { Kalomel } \\
\mathbf{g}\end{array}$ & $\begin{array}{c}\text { Noch } \\
\text { vorhandene } \\
\text { HCOOH } \\
\mathbf{g}\end{array}$ & $\begin{array}{c}\text { Noch } \\
\text { vorhandene } \\
\text { HCOOH } \\
\%\end{array}$ & $\begin{array}{c}\text { Vergoren } \\
\text { HCOOH } \\
\mathbf{g}\end{array}$ & $\begin{array}{c}\text { Vergoren } \\
\text { HCOOH } \\
\%\end{array}$ \\
\hline 1 & 2 & 4,2920 & 0,4390 & 95,39 & 0,0212 & 4,61 \\
2 & 4 & 4,2286 & 0,4133 & 89,80 & 0,0469 & 10,20 \\
3 & 6 & 3,9756 & 0,3885 & 84,42 & 0,0717 & 15,58 \\
4 & 8 & 3,8120 & 0,3725 & 80,95 & 0,0877 & 19,05 \\
5 & 10 & 3,4454 & 0,3367 & 73,17 & 0,1235 & 26,83
\end{tabular}

Die Neutralisation der Nährbouillon wurde mit Hilfe von Phenolphthalein als Indikator durchgeführt; die Kolben wurden nach Methode 2 geimpft. 
Makroskopische Erscheinungen.

Nach 1 Tag: Mittelstarke Trübung, auf der Oberfläche einige isolierte Hautschollen, kein Farbstoff, kein Bodensatz; Kolben sehen gleichmäßig aus.

Nach 2 Tagen: Trübung etwas stärker als am Tage vorher, auf Kolben 6, 7, 8, 9, 10 schwache farblose, nicht zusammenhängende Haut, auf Kolben 5 keine Haut, kein Farbstoff, sehr wenig Bodensatz; Kolben sehen im allgemeinen gleichmäßig aus.

Nach 3 Tagen: Starke Trübung, auf einigen Kolben einige farblose Hautschollen, auf anderen nicht; kein Farbstoff, unangenehmer Geruch, sehr wenig Bodensatz; Kolben sehen im allgemeinen gleichmäßig aus.

Nach 4 Tagen: Sehr starke Trübung, auf der Oberfläche einige farblose isolierte Hautschollen, kein Farbstoff, unangenehmer Geruch, wenig Bodensatz; Kolben sehen gleichmäßig aus.

$\mathrm{Nach} 5$ Tagen: Wie am Tage vorher; Kolben sehen gleichmäßig aus.

Tabelle Nr. 63.

0,4602 g Ameisensäure als Natriumformiat bei $27^{\circ}$.

Bakterium: Bac. Plymouthensis K.G.A.

\begin{tabular}{c|c|c|c|c|c|c}
\hline $\begin{array}{c}\text { Zeit } \\
\text { in } \\
\text { Tagen }\end{array}$ & $\begin{array}{c}\text { Kolben } \\
\text { Nr. }\end{array}$ & Kalomel & $\begin{array}{c}\text { Noch } \\
\text { vorhandene } \\
\text { HCOOH } \\
\mathrm{g}\end{array}$ & $\begin{array}{c}\text { Noch } \\
\text { vorhandene } \\
\text { HCOOH } \\
\%\end{array}$ & $\begin{array}{c}\text { Vergoren } \\
\text { HCOOH } \\
\mathrm{g}\end{array}$ & $\begin{array}{c}\text { Vergoren } \\
\text { HCOOH } \\
\%\end{array}$ \\
\hline 1 & 1 & 4,4894 & 0,4387 & 95,33 & 0,0215 & 4,67 \\
2 & 3 & 4,2194 & 0,4123 & 89,60 & 0,0479 & 10,40 \\
3 & 5 & 3,9684 & 0,3878 & 84,27 & 0,0724 & 15,73 \\
4 & 7 & 3,8082 & 0,3722 & 80,87 & 0,0880 & 19,13 \\
5 & 9 & 3,7086 & 0,3624 & 78,75 & 0,0978 & 21,25
\end{tabular}

Tabelle Nr. 64 .

$0,4602 \mathrm{~g}$ Ameisensäure als Natriumformiat bei $27^{\circ}$.

Bakterium: Bac. Plymouthensis K.G.A.

\begin{tabular}{c|c|c|c|c|c|c}
\hline $\begin{array}{c}\text { Zeit } \\
\text { in } \\
\text { Tagen }\end{array}$ & $\begin{array}{c}\text { Kolben } \\
\text { Nr. }\end{array}$ & Kalomel & $\begin{array}{c}\text { Noch } \\
\text { vorhandene } \\
\text { HCOOH } \\
\mathbf{g}\end{array}$ & $\begin{array}{c}\text { Noch } \\
\text { vorhandene } \\
\text { HCOOH } \\
\%\end{array}$ & $\begin{array}{c}\text { Vergoren } \\
\text { HCOOH } \\
\mathbf{g}\end{array}$ & $\begin{array}{c}\text { Vergoren } \\
\text { HCOOH } \\
\%\end{array}$ \\
\hline 1 & 2 & 4,4756 & 0,4372 & 95,04 & 0,0230 & 4,96 \\
2 & 4 & 4,2086 & 0,4113 & 89,37 & 0,0489 & 10,63 \\
3 & 6 & 3,9550 & 0,3865 & 83,99 & 0,0737 & 16,01 \\
4 & 8 & 3,8164 & 0,3730 & 81,04 & 0,0872 & 18,96 \\
5 & 10 & 3,6914 & 0,3607 & 78,39 & 0,0995 & 21,61
\end{tabular}


Die Neutralisation der Nährbouillon wurde mit Hilfe von Phenolphthalein als Indikator durchgeführt; die Kolben wurden nach Methode 2 geimpft.

Makroskopische Erscheinungen.

Nach 1 Tag: Mittelstarke Trübung, keine Haut, kein Farbstoff, etwas Bodensatz; Kolben sehen gleichmäßig aus.

Nach 2 Tagen: Trübung etwas stärker als am Tage vorher, nur am Rande etwas farblose Haut, kein Farbstoff, etwas Bodensatz; Kolben sehen gleichmäßig aus.

Nach 3 Tagen: Trübung nicht stärker als am Tage vorher, auf der Oberfläche einzelne Hautschollen, am Rande etwas. Haut, auf Kolben 9 etwas mehr Haut als auf 7, 8, 10, kein Farbstoff, in Kolben 9 ziemlich viel Bodensatz; in Kolben 7, 8 und 10 etwas Bodensatz; Kolben sehen im allgemeinen gleichmäßig aus.

Nach 4 Tagen: Kolben 9 bedeutend stärker getrübt als Kolben 10, auf beiden etwas farblose Haut, kein Farbstoff, ziemlich viel Bodensatz; Kolben sehen gleichmäßig aus.

Nach 5 Tagen: Wie am Tage vorher; Kolben sehen ungleichmäßig aus.

Versuchsreihen 63 und 64, welche $\mathrm{zu}$ gleicher Zeit angestellt wurden, wurden 3 Wochen später als Versuchsreihen 61 , 62 , welche ebenfalls gleichzeitig angestellt wurden, durchgeführt.

Vergleich der gefundenen relativen Werte.

\begin{tabular}{rrrrr}
\multicolumn{2}{c}{ Tabelle Nr. 61} & \multicolumn{1}{c}{62} & \multicolumn{1}{c}{63} & \multicolumn{1}{c}{64} \\
\hline 1. Tag & 5,11 & 4,61 & 4,67 & 4,96 \\
2. & 10,89 & 10,20 & 10,40 & 10,63 \\
3. & 15,96 & 15,58 & 15,73 & 16,01 \\
$4 .>$ & 19,32 & 19,05 & 19,13 & 18,96 \\
5. & 27,25 & 26,83 & 21,25 & 21,61
\end{tabular}

Mittlere Tabelle.

\begin{tabular}{|c|c|}
\hline 1. Tag & 4,84 \\
\hline 2. & 10,53 \\
\hline 3. & 15,82 \\
\hline 4. > & 19,12 \\
\hline 5. > & 24,24 \\
\hline
\end{tabular}

Abweichungen der gefundenen von den mittleren Werten.

\begin{tabular}{ccccc} 
Tabelle Nr. 61 & 62 & 63 & 64 \\
\hline 1. Tag $+0,27$ & $-0,23$ & $-0,17$ & $+0,12$ \\
$2 .:+0,36$ & $-0,33$ & $-0,13$ & $+0,10$ \\
$3 .:+0,14$ & $-0,24$ & $-0,09$ & $+0,19$ \\
$4 .:+0,20$ & $-0,07$ & $+0,01$ & $-0,16$ \\
$5 .:++3,01$ & $+2,59$ & $-2,99$ & $-2,63$
\end{tabular}


Die Abweichungen der einzelnen gefundenen Zahlen von den mittleren Werten sind, abgesehen von den Zahlen des 5. Tages, nicht größer als $+0,36 \%$ und $-0,33 \%$. Die Zahlen des 5. Tages, die bei den gleichzeitig angestellten Versuchsreihen 61,62 und 63,64 gut übereinstimmen, differieren untereinander ganz bedeutend; an und für sich hätten also wohl beide Zahlengruppen gleichen Anspruch, als richtig angesehen zu werden. Da aber der Sprung von $19 \%$ auf $27 \%$ viel zu groß ist, dürften wohl die Zahlen 21,25 und 21,61 als passende angenommen werden. Werden die beiden Zahlen des 5. Tages 27,25 und 26,83 nicht berücksichtigt, so wird folgende korrigierte mittlere Tabelle erhalten.

Korrigierte mittlere Tabelle.

$\begin{array}{lr}\text { 1. Tag } & 4,84 \\ 2 . » & 10,53 \\ 3 . » & 15,82 \\ 4 . \$ & 19,12 \\ 5 . \$ & 21,43\end{array}$

Abweichungen der gefundenen von den korrigierten mittleren Werten.

\begin{tabular}{ccccc} 
Tabelle Nr. 61 & 62 & 63 & 64 \\
\hline 1. Tag $+0,27$ & $-0,23$ & $-0,17$ & $+0,12$ \\
2. & $+0,36$ & $-0,33$ & $-0,13$ & $+0,10$ \\
3. & $+0,14$ & $-0,24$ & $-0,09$ & $+0,19$ \\
4. & $+0,20$ & $-0,07$ & $+0,01$ & $-0,16$ \\
5. & $+5,82$ & $+5,40$ & $-0,18$ & $+0,18$
\end{tabular}

Abgesehen von den eingerahmten Zahlen sind die Abweichungen der gefundenen von den korrigierten mittlesen Werten recht klein, sie schwanken zwischen $+0,36$ und $-0,33$.

Menge der während der einzelnen Tage vergorenen Ameisensäure.

(Berechnet aus der korrigierten mittleren Tabelle.) Innerhalb des

1. Tages

2. »

3.

4. \

5. $\gg$ sind vergoren $\mathrm{HCOOH}$

4,84

5,69

5,29

4,30

2,31 
Beiträge zur Biochemie der Mikroorganismen. III.

Vergleich der gefundenen absoluten Werte.

\begin{tabular}{|c|c|c|c|c|}
\hline \multicolumn{2}{|c|}{ Tabelle Nr. 61} & 62 & 63 & 64 \\
\hline 1. Tag & 235 & 212 & 215 & 230 \\
\hline & 501 & 469 & 479 & 489 \\
\hline & 735 & 717 & 724 & 737 \\
\hline 4. & 889 & 877 & 880 & 872 \\
\hline 5. & 1254 & 1235 & 978 & 995 \\
\hline
\end{tabular}

Da die wahre mittlere Tabelle mit der korrigierten mittlejen Tabelle große Ähnlichkeit besitzt, sie weichen nur am 5. Tage voneinander $a b$, soll direkt die korrigierte mittlere Tabelle berechnet werden.

Korrigierte mittlere Tabelle.

$\begin{array}{ll}\text { 1. Tag } & 223 \\ 2 . & 485 \\ 3 . & 728 \\ 4 . & 880 \\ 5 . & 987\end{array}$

Abweichung der gefundenen von den korrigierten mittleren Werten.

\begin{tabular}{lrrrr}
\multicolumn{2}{c}{ Tabelle Nr. 61} & 62 & 63 & 64 \\
\hline 1. Tag & +12 & -11 & -8 & +7 \\
2. & +16 & -16 & -6 & +4 \\
3. & +7 & -11 & -4 & +9 \\
$4 .:$ & +9 & -3 & \pm 0 & -8 \\
5. & +267 & +248 & -9 & +8
\end{tabular}

Abgesehen von den eingerahmten Zahlen von $+0,0267 \mathrm{~g}$ und $+0,0248 \mathrm{~g}$ sind die Abweichungen von den korrigierten mittleren Werten recht klein, sie schwanken zwischen $+0,0016 \mathrm{~g}$ und $-0,0016 \mathrm{~g}$.

Menge der während der einzelnen Tage vergorenen Ameisensäure.

(Berechnet aus der korrigierten mittleren Tabelle.)

$\begin{array}{cr}\text { Innerhalb des } & \text { sind vergoren } \\ \text { 1. Tages } & 223 \\ 2 . & 262 \\ 3 . & 243 \\ 4 . & 152 \\ 5 . & 107\end{array}$

Hoppe-Seyler's Zeitschrift f. physiol. Chemie. LXVII. 
Die erhaltenen Zahlen zeigen eine recht gute Übereinstimmung, nur am 5. Tage kommen größere Abweichungen vor; diese Abweichungen müssen einer Verschiedenartigkeit des Luftzutrittes zugeschrieben werden. Auch aus diesen Versuchsreihen geht wieder mit voller Klarheit hervor, daß zu verschiedenen Zeiten identische Bouillonsorten dargestellt werden können. In 5 Tagen werden $0,0987 \mathrm{~g}=21,43 \%$ Ameisensäure vergoren. Die größte Gärungsintensität liegt innerhalb des 2. Tages; innerhalb dieses Tages werden 0,0262 $\mathrm{g}=5,69 \%$ Ameisensäure vergoren; nach diesem Tag fällt die Gärungsintensität sehr allmählich $\mathrm{ab}$ und ist nach 5 Tagen noch nicht beendet.

\section{Vergleich der bei den einzelnen Temperaturen erhaltenen Zahlen.}

Die größte Gärungsintensität liegt bei allen Temperaturen innerhalb des 2. Tages. An diesem Tage werden vergoren bei $17^{\circ} 0,0171 \mathrm{~g}=3,68 \%$

» $21^{\circ} 0,0237 »=5,09 \%$

» $27^{\circ} 0,0262 »=5,69 \%$

Nach 5 Tagen sind vergoren

$$
\begin{aligned}
\text { bei } 17^{\circ} 0,0635 \mathrm{~g}=13,80 \% \\
\gg 21^{\circ} 0,0810 »=17,60 \% \\
» 27^{\circ} 0,0987 »=21,43 \%
\end{aligned}
$$

Bei $21^{\circ}$ wird mehr Ameisensäure vergoren als bei $17^{\circ}$ und bei $27^{\circ} \mathrm{mehr}$ als bei $21^{\circ}$. Die Differenz zwischen $17^{\circ}$ und $21^{\circ}$ beträgt $0,0175 \mathrm{~g}=3,80 \%$ und die zwischen $21^{\circ}$ und $27^{\circ} 0,0177 \mathrm{~g}=3,83 \%$. Der Sprung von $17^{\circ}$ auf $21^{\circ}$ (Unterschied $4^{\circ}$ ) ist ebensogroß, wie der zwischen $21^{\circ}$ und $27^{\circ}$ (Untersehied $6^{\circ}$ ).

Vergleich von Bac. Plymouth. Kràl mit Bac. Plymouth. K. G. A.

Nach 5 Tagen sind vergoren:

\section{Kràl}

$17^{\circ}$
K. G. A.

$$
0,0635 \mathrm{~g}=13,80 \%
$$


Beiträge zur Biochemie der Mikroorganismen. III.

$\begin{array}{lll}0,1176 \mathrm{~g}=25,55 \% & \mathbf{2 1}^{\circ} & 0,0810 \mathrm{~g}=17,60 \% \\ 0,1273 \mathrm{~g}=27,65 \% & \mathbf{2 7}{ }^{\circ} & 0,0987 \mathrm{~g}=21,43 \%\end{array}$

Aus diesen Zahlen geht hervor, daß Bac. Plymouth. Kràl nach 5 Tagen bedeutend mehr Ameisensäure zu vergären vermag als Bac. Plymouth. K.G.A.

Die Differenz beträgt bei

$$
\begin{array}{ll}
17^{\circ} & 0,0138 \mathrm{~g}=3,02 \% \\
21^{\circ} & 0,0366 \mathrm{~g}=7,95 \% \\
27^{\circ} & 0,0286 \mathrm{~g}=6,22 \%
\end{array}
$$

Während bei Bac. Plymouth. K.G.A. die Differenzen zwischen 17 und $21^{\circ}$ und zwischen 21 und $27^{\circ}$ gleich groß sind, ist die Differenz bei Bac. Plymouth. Kràl zwischen 17 und $21^{\circ}$ bedeutend größer als die zwischen 21 und $27^{\circ}$. Hieraus läßt sich wohl mit ziemlicher Sicherheit schließen, daß die Optimaltemperatur für die Vergärung der Ameisensäure durch Bac. Plymouth. Kràl eine andere ist, als die für Bac. Plymouth. K.G.A.

Diese Zahlen geben natürlich noch kein endgültiges Bild von der Verschiedenheit der beiden Stämme, da die Zahlen mittlere Werte sind und die einzelnen Zahlen teilweise recht schlecht miteinander übereinstimmen. Wegen dieser Ungleichmäßigkeit der erhaltenen Zahlen ist es auch vorläufig zwecklos, die Werte von Tag zu Tag zu vergleichen, um eventuell Gesetzmäßigkeiten zu finden; dies muß verschoben werden, bis eine absolute Gleichmäßigkeit des Nährmaterials und der Luftzufuhr erreicht ist und dadurch eine bessere Übereinstimmung der Zahlen erreicht wird.

Die Verschiedenheit der erhaltenen Werte ist jedenfalls auf eine Verschiedenheit des physiologischen Zustandes der beiden Bakterienstämme zurückzuführen. Vergleicht man die bei beiden Stämmen beobachteten makroskopischen Erscheinungen miteinander, so wird man finden, daß sich im allgemeinen dasselbe Bild ergibt. Aus den erhaltenen makroskopischen Bildern würde man wohl kaum auf eine physiologische Verschiedenheit der beiden Stämme schließen können, während 
296 Fr. Kutscher, Zur Arbeit von F. Malengreau u. A. Lebailly.

die erhaltenen Zahlen die physiologische Verschiedenheit beweisen. Hier sieht man deutlich, daß nur durch eine quantitative chemische Analyse solche physiologische Verschiedenheiten aufgedeckt werden können, welche bei der gewöhnlichen bakterischen Beobachtung nicht zutage treten. Auch läßt sieh durch die quantitative chemische Analyse eine exakt zahlenmäßige Beziehung finden, deren Aufstellung mit Hilfe der üblichen bakteriologischen Arbeitsweisen unmöglich ist. Nur läßt sich in unserem Falle diese zahlenmäßige Beziehung noch nicht mit Sicherheit aufstellen, da vorläufig die Zahlen noch zu wenig miteinander übereinstimmen. Aber wenn die diese Verschiedenheiten bedingenden Faktoren erst einmal ausgeschaltet sind, werden die Beziehungen mit aller Klarheit hervortreten.

\section{Notiz zu der Arbeit von F. Malengreau und A. Lebailly: Über die synthetischen Homocholine.}

(Diese Zeitschrift, Bd. LXVII, Heft 1.)

$$
\text { Von }
$$

Fr. Kutscher.

(Der Redaktion zugegangen am 22. Juni 1910.)

In meinem Laboratorium hat bereits vor längerer Zeit Herr Berlin die Homocholine synthetisch dargestellt.

Zum Vergleich hat er auch größere Mengen von Neosin aus Krabbenextrakt zu gewinnen versucht. Die letztere Arbeit war sehr schwierig und zeitraubend und hat deshalb bisher eine Veröffentlichung seiner Resultate hintangehalten. Ich bitte, dieses Gebiet noch kurze Zeit meinem Laboratorium überlassen $\mathrm{zu}$ wollen. 